\title{
Reconstructing extreme AMOC events through nudging of the ocean surface: a perfect model approach
}

\author{
Pablo Ortega $^{1,2}\left(\right.$ Cric Guilyardi $^{1,2} \cdot$ Didier Swingedouw $^{3} \cdot$ Juliette Mignot $^{1,4} \cdot$ \\ Sébastien Nguyen ${ }^{1}$
}

Received: 27 July 2016 / Accepted: 3 January 2017

(c) The Author(s) 2017. This article is published with open access at Springerlink.com

\begin{abstract}
While the Atlantic Meridional Overturning Circulation (AMOC) is thought to be a crucial component of the North Atlantic climate, past changes in its strength are challenging to quantify, and only limited information is available. In this study, we use a perfect model approach with the IPSL-CM5A-LR model to assess the performance of several surface nudging techniques in reconstructing the variability of the AMOC. Special attention is given to the reproducibility of an extreme positive AMOC peak from a preindustrial control simulation. Nudging includes standard relaxation techniques towards the sea surface temperature and salinity anomalies of this target control simulation, and/or the prescription of the wind-stress fields. Surface nudging approaches using standard fixed restoring terms succeed in reproducing most of the target AMOC variability, including the timing of the extreme event, but systematically underestimate its amplitude. A detailed analysis of the AMOC variability mechanisms reveals that the underestimation of the extreme AMOC maximum comes from a deficit in the formation of the dense water masses in the main convection region, located south of Iceland in the model. This issue is largely corrected after introducing a novel surface nudging approach, which uses a varying restoring coefficient that is proportional to the simulated
\end{abstract}

Pablo Ortega

p.ortega@reading.ac.uk

1 LOCEAN/IPSL, Sorbonne Universités (UPMC)-CNRS-IRDMNHN, 4 place Jussieu, 75005 Paris, France

2 NCAS-Climate, University of Reading, Reading, UK

3 EPOC, Université Bordeaux, Bordeaux, France

4 Climate and Environmental Physics and Oeschger Centre for Climate Change Research, University of Bern, Bern, Switzerland mixed layer depth, which, in essence, keeps the restoring time scale constant. This new technique substantially improves water mass transformation in the regions of convection, and in particular, the formation of the densest waters, which are key for the representation of the AMOC extreme. It is therefore a promising strategy that may help to better constrain the AMOC variability and other ocean features in the models. As this restoring technique only uses surface data, for which better and longer observations are available, it opens up opportunities for improved reconstructions of the AMOC over the last few decades.

Keywords Decadal variability · Atlantic Meridional Overturning Circulation · Surface nudging $\cdot$ Coupled general circulation model

\section{Introduction}

The ocean circulation, and in particular the Atlantic Meridional Overturning Circulation (AMOC), is an important driver of climate variability in the North Atlantic (Delworth et al. 1993; McCarthy et al. 2015) that can modulate regionally the global warming signal. Rapid and large decadal fluctuations in the AMOC strength emerge frequently in models as a result of internal climate variability, showing also important widespread climate impacts (Allison et al. 2014). However, most of these results refer to the model world. Our current knowledge of the real AMOC variability is rather limited due to the lack of long adequate observations. Continuous measurements available since 2004 thanks to the RAPID monitoring array (Cunningham et al. 2007), located along the $26^{\circ} \mathrm{N}$ latitudinal transect, report a slight decreasing trend during the last 10 years (Smeed et al. 2014). A complementary altimetry-based 
reconstruction of the AMOC along the OVIDE section (from Greenland to Portugal) also shows a decline in its strength between 1993 and 2010 (Mercier et al. 2015). However, both records are still too short to determine if this decadal trend emerges in response to global warming, or if it is compatible with natural ocean fluctuations.

It is therefore of paramount importance to explore different ways of reconstructing AMOC variability back in time, and use them to make inferences about its characteristic time scales and variability drivers. One can distinguish four major ways of estimating past AMOC variability. First, the use of proxy records from sedimentary sources, usually sensitive to the deep ocean currents (e.g. McManus et al. 2004; Kissel et al. 2013; Thornalley et al. 2013). These estimates are, however, localised and challenging to interpret. Second, reconstructions can be based on well-established physical equations (Duchez et al. 2014; Frajka-Williams 2015; McCarthy et al. 2015) linking AMOC variability to that of other variables more easily observed. For example, the thermal wind relationship allows to compute the meridional geostrophic transport from zonal density gradients (Marotzke et al. 1999), and constitutes the conceptual framework for the RAPID operational array. The most important shortcoming of this approach is the necessity of long and widespread observations from hardly accessible regions like the deep ocean. The third method relies on the identification of significant statistical relationships between the AMOC and other oceanic and atmospheric variables, such as the sea surface temperature and height (Latif et al. 2004; Zhang 2008; Willis 2010; Duchez et al. 2016), the deep Labrador sea water densities (Robson et al. 2014), or the cumulative effect of heat fluxes in the deep convection regions (Ortega et al. 2011). However, these covariances have been mostly established using models, which have inevitable biases that may compromise the reality of these inferred relationships. Besides, direct observations of the AMOC are still too short and can only be used to identify significant relationships at the monthly timescale (Duchez et al. 2016), which do not necessarily hold at decadal and longer timescales. Finally, climate models can also be used to constrain the past AMOC evolution, by assimilating the observed variability of relevant climate quantities (e.g. Huck et al. 2008; Swingedouw et al. 2013; Shaffrey et al. 2016). Yet, while products assimilating surface data (Reichler et al. 2012) show encouraging agreement in their representation of AMOC variability, other ocean reanalyses including subsurface data reveal large discrepancies (Karspeck et al. 2015), thus casting doubt on the efficiency of current assimilation techniques, which are not specifically designed to reconstruct AMOC variability.

This paper deals with this latter approach. It explores new ways of assimilating observations in the models and investigates their relevance to reproduce the associated
AMOC variability. Such reconstructions are also essential for decadal prediction purposes as they provide the necessary initial ocean conditions for hindcasts and forecasts.

To date, different modelling groups have implemented and explored a variety of reconstruction protocols, following various techniques and model setups (Meehl et al. 2014). One standard approach is to apply a simple Newtonian relaxation (or nudging) towards one or several observational fields, as opposed to more complex assimilation techniques used, e.g., in ocean reanalyses (Balmaseda et al. 2015). In both cases, one important choice to be made is the vertical extent of the variables that are initialised-involving either surface (e.g. Ray et al. 2015; Servonnat et al. 2015) or three-dimensionnal (3D) ocean fields (e.g. Pohlmann et al. 2009; Tatebe et al. 2012). Subsurface nudging is probably more efficient in constraining the zonal and meridional density gradients which, through geostrophic balance, influence the ocean circulation (e.g. Huck et al. 2008; Sévellec and Fedorov 2013). Widespread reliable observations at these levels are nevertheless only globally available since $2000 \mathrm{AD}$, with the deployment of ARGO profiling floats. Before this year, observations of the deep ocean are subject to large uncertainties that affect the reliability of assimilation in regions of key importance for the AMOC like the Gulf Stream Extension or the Labrador Sea (Turpin et al. 2016). This loss of reliability is in line with the reported lack of consistency among ocean reanalyses regarding the temperature evolution below $500 \mathrm{~m}$ (Ray et al. 2015), the AMOC (Karspeck et al. 2015) and the subpolar gyre (Born et al. 2015). These limitations are a strong motivation to use surface restoring, for which quality records extend further back in time.

Reconstructing the annual AMOC variability through surface restoring relies on the assumption that the ocean interior can be initialised through a proper representation of relevant processes forced by the ocean surface, like deep ocean convection and ventilation, which show important variability at shorter timescales (monthly and seasonal). Ocean surface restoring is however sensitive to the quality of the model, and in particular to its correct simulation of internal variability. Yet, this approach has the potential to exploit the highest reliability and best temporal and spatial coverage of surface observations. Surface relaxation is classically done by adding a restoring heat flux component $\gamma_{\mathrm{T}}\left(S S T_{\text {model }}-S S T_{\text {target }}\right)$ to the SST conservation equation. Thus, a key choice is the strength of the relaxation term $\gamma_{\mathrm{T}}$ to be applied. While measurements of the oceanic heat flux feedback estimate $\gamma_{\mathrm{T}}$ to be close to $-40 \mathrm{~W} / \mathrm{m}^{2} / \mathrm{K}$ (Barnier et al. 1995; Frankignoul and Kestenare 2002), many nudging studies have considered substantially larger restoring terms (6-60 times larger; e.g. Luo et al. 2005; Pohlmann et al. 2009; Dunstone and Smith 2010) in order to constrain the simulated temperatures more closely to observations. 
However, there are several drawbacks to using large $\gamma_{T}$ values. One is the constant forcing of overly high restoring heat fluxes in regions with strong model biases (ISSTmodel $-\mathrm{SST}_{\text {target }} \mathrm{I} \gg 0$ ), such as in the Gulf Stream, creating spurious water masses that can interfere with the internal ocean variability. Other significant issues include the tampering with key high-frequency ocean-atmosphere interactions (Christophe Cassou pers. comm.) as well as with subsurface stratification. Some initial tests within our group also suggest that excessively large restoring coefficients can also overwhelm the salt-advection feedback first described by Stommel (1961), which is key to guarantee AMOC stability. It is therefore of great importance to explore new methodologies that could overcome or minimise these effects. Initialized experiments can additionally or alternatively assimilate salinity (Servonnat et al. 2015), sea ice (e.g. Caya et al. 2010), land soil moisture (e.g. Han et al. 2012), as well as different atmospheric fields, such as wind-stress (Ding et al. 2013; Thoma et al. 2015) or the 3D winds (Stewart and Haine 2013). Likewise, assimilation can be applied in ocean-only (e.g. Yeager et al. 2012) or atmosphere-ocean coupled model (e.g. Yang et al. 2013; Servonnat et al. 2015) configurations.

Wind-nudging alone can also potentially reconstruct ocean dynamics, and in particular the AMOC variability, both by directly reproducing the wind-driven circulation, and also indirectly via the wind contribution to turbulent fluxes (Large and Yeager 2009). Thoma et al. (2015) have shown that wind-stress anomaly assimilation in partiallycoupled simulations is able to reconstruct most of the observed AMOC variability at $26^{\circ} \mathrm{N}$ in the $2005-2012$ period, although it is not clear if the skill goes beyond the reconstruction of the Ekman transport component. Matei et al. (2012) also report multiyear predictive skill for the monthly AMOC at $26^{\circ} \mathrm{N}$ in a set of experiments with the ECHAM5/MPI-OM, initialized from an ensemble of MPIOM ocean-only simulations forced with reanalyzed atmospheric data. However, the validity of these results has been called into question, given the limited forecast evaluation period considered and the inclusion of the annual cycle in their assessment (Vecchi et al. 2012). Indeed, robust forecast skill estimates would require of long reference observational records that are still not available.

A first step to evaluate the best methods to reconstruct past AMOC variations is the use of perfect model frameworks (Boer 2004). By using the models as a surrogate for the real world, where the climate system is perfectly known and therefore observational limitations do not apply, it is possible to test the potential predictability of the AMOC and the performance of different initialization techniques in a longer term perspective. For example, a perfect model study with the IPSL-CM5A-LR model suggests that most of the simulated AMOC variability in a target control simulation can be correctly reconstructed when both sea surface temperature (SST) and salinity (SSS) nudging are applied (Servonnat et al. 2015), despite issues in reproducing the amplitude of the largest AMOC variations. Likewise, Zhang et al. (2010) have shown that the ability to properly characterise the AMOC variability is substantially improved when both temperature and salinity are assimilated down to $2000 \mathrm{~m}$.

Other perfect model studies (Dunstone and Smith 2010; Counillon et al. 2014) also suggest potential predictability of the AMOC variability for lead times of up to a decade, after initializing the ocean through subsurface temperature and salinity assimilation, or via SST-only assimilation. AMOC predictability has been shown to depend on the initial ocean state (Msadek et al. 2010), with better skill generally associated with hindcasts starting from extreme (maxima and minima) AMOC states (Collins et al. 2006; Persechino et al. 2013). This conditional skill, estimated in studies that assume perfectly known initial conditions, can be only achieved in the historical context if the initial ocean state is well constrained, in particular during the extreme AMOC states.

Using a perfect model approach, this study explores the ability of different reconstruction techniques to reproduce the simulated AMOC variability in a target period of a preindustrial control simulation, specifically selected to include an extreme positive AMOC event. Because of its potential to produce longer reconstructions, only surface nudging is used and several types of restoring coefficients are explored. This study also addresses the sensitivity of the results to the starting conditions used in the nudged experiments. The paper is organised as follows: the model, methodology and experimental setup are described in Sect. 2. Section 3 analyses the performance of the standard reconstruction techniques, and Sect. 4 investigates the reasons for the extreme AMOC peak, and its underestimation in the associated nudged experiments. Section 5 builds on the previous findings to propose and validate a novel nudging approach, based on a variable restoring coefficient that is proportional to the mixed layer depth. This new technique overcomes the major limitations of classic nudging approaches and emerges as an ideal compromise between weak and strong restoring terms. Finally, the major findings of the study are summarized and discussed in Sect. 6.

\section{Model and experimental setup}

\subsection{Model configuration}

This study is based on the Institut Pierre Simon Laplace coupled model IPSL-CM5A-LR, a standard CMIP5 configuration presented and described in details in Dufresne 
et al. (2013). This is the same model version used in previous perfect model studies focused both on surface nudging techniques for ocean initialisation (Servonnat et al. 2015) and potential predictability of the AMOC (Persechino et al. 2013), and has been extensively used in other studies analysing mechanisms of natural decadal variability in the North Atlantic (Escudier et al. 2013; Ortega et al. 2015), as well as evaluating historical decadal prediction skill in the ocean through surface nudging (Swingedouw et al. 2013; Mignot et al. 2015). Using IPSL-CM5A-LR thus allows us to benefit from this in-depth expertise of the model, and also from its relatively cheap computational cost, ideal to run several sets of ensemble experiments. The model is composed of the atmospheric component LMDZ5A (Hourdin et al. 2013) at the CMIP5 $96 \times 96$ resolution (i.e. $\left.3.75^{\circ} \times 1.875^{\circ}\right)$, and the oceanic component NEMOv3.2 (Nucleus of European Modelling of the Ocean, Madec 2008), itself composed of the ocean model OPA9 and the sea ice component LIM2 (Fichefet and Maqueda 1997), both using an ORCA2.0 tripolar grid that has an averaged horizontal resolution of $2^{\circ} \times 2^{\circ}$ refined to $0.5^{\circ}$ in the tropics. The respective vertical resolution corresponds to 39 levels in the atmosphere and 31 in the ocean. Other components also included in IPSL-CM5A-LR are the land-surface model ORCHIDEE (Krinner et al. 2005), and the biogeochemistry module PISCES (Aumont and Bopp 2006), which is integrated in NEMO. Various studies describing the different components and several aspects of the model performance are gathered in a special issue of Climate Dynamics (http://link.springer.com/journal/382/40/9/), including an assessment of the oceanic module in the coupled model configuration (Mignot et al. 2013).

\subsection{Methodology and experimental protocol}

Following the Servonnat et al. (2015) approach, this analysis is carried out in the framework of the 1000year CMIP5 preindustrial control simulation, described and analysed in Escudier et al. (2013). A subset of this simulation (CTRL from now on)-characterised by the occurrence of an extreme AMOC maximum (in the simulation year 2070)—constitutes the target trajectory and provides the surface boundary conditions (for all the model components) used to drive the nudging experiments in the present perfect model study. Sensitivity to the initial conditions is explored by considering three different initial states, corresponding to weak, moderate and strong AMOC conditions (i.e. 5.8, 9.5 and 13.4 Sv at $48^{\circ} \mathrm{N}$, respectively) chosen outside of the target period in the control simulation. An estimate of the best potential initialisation achievable by each nudging technique is explored in additional experiments using exact ocean starting conditions from CTRL, representing therefore the correct initial state for each of the relevant AMOC precursors (see Fig. 1, and Sect. 4 for more details). These simulations are expected to follow a different trajectory than CTRL because they were run in a different machine with different round-off errors, and also because of the restoring terms introduced by the nudging. The bulk of the experiments is started 15 years before the AMOC target maximum, to provide sufficient time for these AMOC precursors to be initialised. Three standard surface nudging protocols are implemented and analysed.

The first initialization approach (Wind15P) is based solely on the prescription of the wind-stress components $\tau_{\mathrm{x}}$ and $\tau_{\mathrm{y}}$ that are seen by the ocean, each of them according to the formula:

$\tau=(1-\alpha) \tau_{\text {model }}+\alpha \times \tau_{\text {target }}$,

where $\tau_{\text {model }}$ is the simulated wind-stress in the nudged experiment, $\tau_{\text {target }}$ the wind-stress in the target CTRL period and $\alpha$ is the restoring coefficient, whose value is 1 poleward of $20^{\circ} \mathrm{N} / \mathrm{S}$, zero between $10^{\circ} \mathrm{S}$ and $10^{\circ} \mathrm{N}$, and follows a smooth step-like function in the $10^{\circ} \mathrm{S}-20^{\circ} \mathrm{S}$ and $10^{\circ} \mathrm{N}-20^{\circ} \mathrm{N}$ latitudinal bands. The tropical band is excluded to avoid interfering with the strong tropical air-sea coupling. This restoring approach is expected to reconstruct the AMOC by representing the wind-driven circulation and its effect on the transport of (i.e. climatological) sea surface temperature and salinity. The key AMOC precursor in this case is the East Greenland Current, which has an important Ekman-driven contribution in IPSL-CM5A-LR (Escudier et al. 2013, Fig. 1).

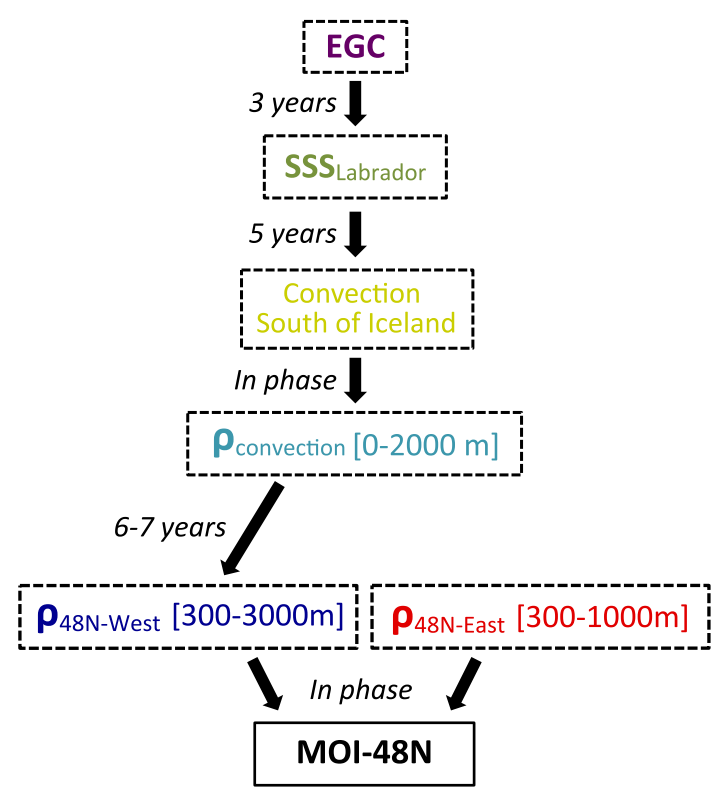

Fig. 1 Schematic of the AMOC precursors in the ISPL-CM5A-LR model, as identified in Escudier et al. (2013) and Ortega et al. (2015). The estimated leading times are indicated in the arrows 
The second methodology (SSTS15P) corresponds to the nudging strategy showing the best performance in the analysis of Servonnat et al. (2015) in terms of AMOC reconstruction. It consists of applying a joint SST and SSS restoring in the sea-ice free regions. This regional constraint avoids problems related to the occurrence of spurious buoyancy forcing and convection near the sea-ice border. A standard nudging approach is applied on a daily basis by adding a Haney fixed restoring term $\gamma^{\text {fix }}$ to the conservation of heat and salt, as described by the following equations:

$\frac{\partial S S T}{\partial t}=\cdots+\frac{\gamma_{T}}{\rho_{0} C_{p} h}\left(S S T_{\text {model }}-S S T_{\text {target }}\right)$,

$\frac{\partial S S S}{\partial t}=\cdots+\frac{\gamma_{S}}{h}\left(S S S_{\text {model }}-S S S_{\text {target }}\right)$

where $\rho_{0}$ is the mean ocean density, $c_{p}$ the specific heat of the ocean, $h$ the depth of the associated surface layer, and $\gamma_{\mathrm{T}}$ and $\gamma_{\mathrm{S}}$ the restoring coefficients controlling the strength of the nudging in temperature and salinity. $\mathrm{SST}_{\text {target }}$ and $\mathrm{SSS}_{\text {target }}$ are linearly interpolated at the daily resolution from the target monthly-resolved CTRL data. As in our previous studies (Swingedouw et al. 2013; Servonnat et al. 2015), these restoring terms are fixed respectively to $-40 \mathrm{~W} / \mathrm{m}^{2} / \mathrm{K}$ and $-864 \mathrm{~mm} /$ day, two estimates corresponding both to a relaxation timescale of about 60 days over a $50 \mathrm{~m}$ deep mixed layer. While the first is based on the observed temperature/heat flux relationships, the second has no physical basis and is chosen to yield the same relaxation timescale as in the first. For further discussion on the magnitude of the restoring coefficient, please refer to Servonnat et al. (2015). This nudging approach is expected to be more effective than Wind15P in initialising the AMOC, as the combined restoring of both SSS and SST enables a direct representation of the target North Atlantic deep convection region (Servonnat et al. 2015), which is

largely driven in the model by SSS changes in the Labrador Sea (Fig. 1). Finally, the third set of nudging experiments includes the prescription of wind-stress fields combined with the restoring in SST and SSS (SSTSWind15P).

As indicated above, an ensemble of four experiments is performed for each of these approaches, using different initial conditions. Several additional experiments are conducted beyond this basic protocol in order to assess the consistency of our results and identify potential performance improvements. These include longer simulations, which start 25 years before the occurrence of the peak to provide a longer time for the deeper ocean to be initialised. We also develop a novel nudging strategy based on a variable restoring term $\gamma^{\mathrm{var}}$, detailed in Sect. 5. The complete set of model experiments performed and analysed in this study is summarised in Table 1.

\subsection{Assessment of nudging performance}

Several metrics are used throughout the analysis to assess different aspects of the performance of the nudging techniques. For any given variable $X$, the average distance to the target CTRL variability is evaluated with the ensemble mean absolute error $(\mathrm{AE})$, which is computed as follows:

$A E(X)=\frac{1}{m} \sum_{i=1}^{m}\left|X_{C T R L}-X_{i}\right|$,

where $i$ is an index that goes through the four different ensemble members.

Also, the sensitivity to the initial ste is explored using the mean intra-ensemble distance (IED), which averages the mean distance between any two ensemble members $i, j$ as described in the following equation:

$$
\operatorname{IED}(X)=\frac{1}{12} \sum_{i=1}^{4} \sum_{\substack{j=1 \\ j \neq i}}^{4}\left|X_{j}-X_{i}\right| .
$$

\begin{tabular}{|c|c|c|c|c|}
\hline Experiment family & SST and SSS restoring & $\begin{array}{l}\text { Prescribed wind- } \\
\text { stress }\end{array}$ & $\begin{array}{l}\text { Initial date (prior to the } \\
\text { peak) (years) }\end{array}$ & Starting conditions \\
\hline Wind15P & No & Yes & 15 & $\mathrm{~S}, \mathrm{~W}, \mathrm{M}, \mathrm{E}$ \\
\hline SSTS15P & Fixed $\left(\gamma^{\mathrm{fix}}\right)$ & No & 15 & $\mathrm{~S}, \mathrm{~W}, \mathrm{M}, \mathrm{E}$ \\
\hline SSTS25P & Fixed $\left(\gamma^{\mathrm{fix}}\right)$ & No & 25 & $\mathrm{M}, \mathrm{E}$ \\
\hline SSTSWind15P & Fixed $\left(\gamma^{\text {fix }}\right)$ & Yes & 15 & $\mathrm{~S}, \mathrm{~W}, \mathrm{M}, \mathrm{E}$ \\
\hline SSTSWind25P & Fixed $\left(\gamma^{\mathrm{fix}}\right)$ & Yes & 25 & $\mathrm{M}, \mathrm{E}$ \\
\hline SSTSWind15Pvg & Mixed-layer depth dependent $\left(\gamma^{\mathrm{var}}\right)$ & Yes & 15 & $\mathrm{M}, \mathrm{E}$ \\
\hline SSTSWind25Pvg & Mixed-layer depth dependent $\left(\gamma^{\text {var }}\right)$ & Yes & 25 & $\mathrm{M}, \mathrm{E}$ \\
\hline SSTS25Pvg & Mixed-layer depth dependent $\left(\gamma^{\mathrm{var}}\right)$ & No & 25 & $\mathrm{M}, \mathrm{E}$ \\
\hline
\end{tabular}

Table 1 Summary of the different families of nudging experiments

Up to four different experiments have been performed for each experiment family, differing by the starting conditions considered: strong (S), weak $(\mathrm{W})$, moderate $(\mathrm{M})$ and exact $(\mathrm{E})$. In all experiments, the restoring is only applied over sea ice-free regions. When applied, wind stress is prescribed everywhere out of the Tropics $\left(10^{\circ} \mathrm{S}-10^{\circ} \mathrm{N}\right)$ 
To highlight extreme variability in the different indices, most figures include the thresholds of the 5th and 95th percentiles from the 1000-year long control simulation (e.g. lower and upper horizontal dashed lines in Fig. 2). Values exceeding these thresholds will be referred to as extreme. Besides, in some cases, the extreme nature of the peak in CTRL and the nudged simulations will be quantified as the number of standard deviations away from the 1000-year control climatological mean (Table 2).
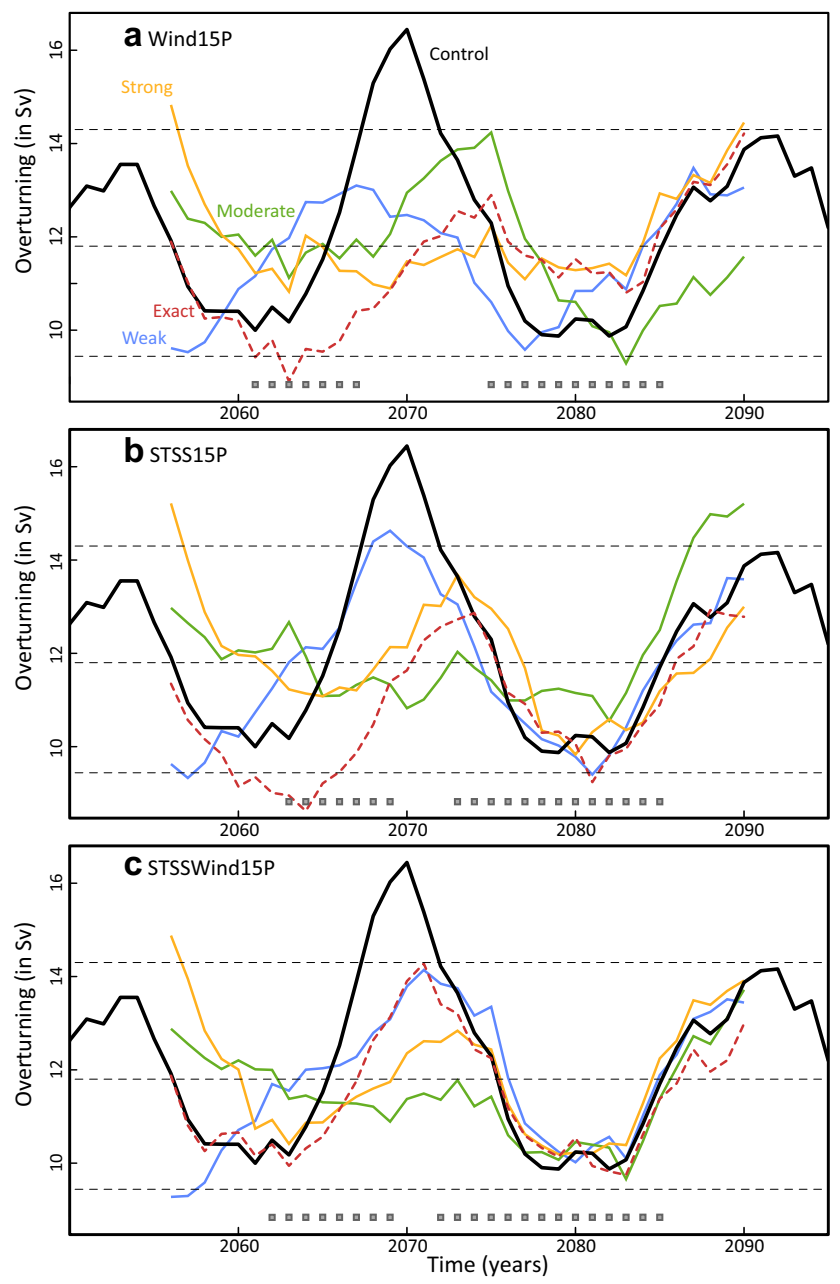

Fig. 2 Evolution of the maximum AMOC value at $48^{\circ} \mathrm{N}$ after the Ekman component is removed (MOI-48N) in CTRL and three ensembles of nudged simulations: a WindOnly, b SurfaceTS and c SurfaceTSWind. Each ensemble consists of four individual members initialized from weak (blue), moderate (green), strong (orange) and exact (dotted red) AMOC starting conditions taken from CTRL. Horizontal dashed lines mark the climatological mean and the associated 5th and 95th percentiles of CTRL. Grey squares at the bottom of each panel highlight the centre of a 11-year long sliding window in which CTRL and the ensemble mean of the four experiments correlate significantly at the $95 \%$ confidence level

\section{Performance of the standard nudging techniques}

The reference meridional overturning index to evaluate AMOC initialisation in the different nudged experiments is defined as the annual local vertical maximum of the AMOC streamfunction at $48^{\circ} \mathrm{N}$ (MOI- $48 \mathrm{~N}$; Fig. 1). This corresponds to the latitude where the full AMOC streamfunction and the northward thermal-wind transport reach their climatological maximum in the model (Ortega et al. 2015). Since our interest lies in the thermohaline-driven component of the AMOC, the Ekman transport signal is removed from the MOI- $48 \mathrm{~N}$ index.

Figure 2 shows how the MOI- $48 \mathrm{~N}$ is reconstructed by the different standard nudging techniques. Wind-stress alone is insufficient to reproduce the main positive peak, at least consistently and at the right time, although all the Wind15P individual experiments do represent the second peak in year 2090. The poor representation of the first maximum is confirmed by the large AE values in Fig. 3a during the decade centered in the peak. These errors are slightly reduced in the SSTS15P ensemble, which also better represents the timing of the peak (Fig. 2). The amplitude is nevertheless still clearly underestimated. In both Wind15P and SSTS15P ensembles, the initial state has an impact on the reconstruction. For example, in SSTS15P, there is a stark contrast between the experiments started from weak and moderate AMOC conditions, the first showing a clear MOI-48N maximum that crosses the 95th percentile, and the second missing completely the peak. The same occurs when comparing the experiments started from moderate and strong AMOC conditions in Wind15P. Interestingly, the first peak is also misrepresented by the experiments using exact initial conditions, thereby highlighting important limitations in these standard nudging approaches to capture the extreme nature of the peak.

Adding wind-stress to SST and SSS nudging does not lead to any improvement regarding the amplitude of the first peak (Fig. 2c), but the sensitivity to initial AMOC conditions appears to be substantially reduced, most notably from year 2080 onwards (Fig. 3c). Also, this is the only configuration for which, as one would expect, the experiment starting from exact ocean conditions reproduces the AMOC peak more accurately (in terms of timing and magnitude, Fig. 2). This, together with the reduced IED in the SSTSWind15P ensemble, suggests that this combined technique is the less sensitive to the initial state. The best overall performance of SSTSWind15P is confirmed when the different metrics in Fig. 3 are accumulated in time. As compared to the other approaches, this combined nudging technique reduces by at least $20 \%$ the ensemble mean $\mathrm{AE}$ and by $30 \%$ the mean IED (Fig. 3d) after 45 years of integration. 
Table 2 Summary of magnitude and timing of the peaks in the ocean precursors related to the extreme maximum in MOI-48N

\begin{tabular}{llll}
\hline Variables & Long-term control mean & $\begin{array}{l}\text { Long-term control standard } \\
\text { deviation (SD) }\end{array}$ & $\begin{array}{l}\text { Magnitude of the peak (SDs } \\
\text { above/below the mean) }\end{array}$ \\
\hline EGC & $5.3 \mathrm{~Sv}$ & $\begin{array}{l}\text { Timing } \\
\text { of the } \\
\text { peak }\end{array}$ \\
SSS $_{\text {Labrador }}$ & $33.2 \mathrm{psu}$ & $0.5 \mathrm{~Sv}$ & $4.1 \mathrm{~Sv}(2.4 \mathrm{SDs})$ \\
MLD $_{\text {SouthernRegion }}$ & $278 \mathrm{~m}$ & $0.4 \mathrm{psu}$ & $34.2 \mathrm{psu}(2.5 \mathrm{SDs})$ \\
$\rho_{\text {convection }[0-1000 \mathrm{~m}]}$ & $27.81 \mathrm{~kg} / \mathrm{m}^{3}$ & $134 \mathrm{~m}$ & $581 \mathrm{~m}(2.3 \mathrm{SDs})$ \\
$\rho_{\text {convection }[1000-2000 \mathrm{~m}]}$ & $27.89 \mathrm{~kg} / \mathrm{m}^{3}$ & $0.04 \mathrm{~kg} / \mathrm{m}^{3}$ & $27.90 \mathrm{~kg} / \mathrm{m}^{3}(2.3 \mathrm{SDs})$ \\
$\rho_{48 \mathrm{~N}-\text { East }[300-1000 \mathrm{~m}]}$ & $27.66 \mathrm{~kg} / \mathrm{m}^{3}$ & $0.01 \mathrm{~kg} / \mathrm{m}^{3}$ & $27.92 \mathrm{~kg} / \mathrm{m}^{3}(3 \mathrm{SDs})$ \\
$\rho_{48 \mathrm{~N}-W e s t ~[300-3000 \mathrm{~m}]}$ & $27.90 \mathrm{~kg} / \mathrm{m}^{3}$ & $0.01 \mathrm{~kg} / \mathrm{m}^{3}$ & $27.64 \mathrm{~kg} / \mathrm{m}^{3}(2 \mathrm{SDs})$ \\
$\rho_{48 \mathrm{~N}-W e s t ~[2000-3000 \mathrm{~m}]}$ & $27.93 \mathrm{~kg} / \mathrm{m}^{3}$ & $0.008 \mathrm{~kg} / \mathrm{m}^{3}$ & $27.92 \mathrm{~kg} / \mathrm{m}^{3}(2.5 \mathrm{SDs})$ \\
MOI-48N & $11.8 \mathrm{~Sv}$ & $0.007 \mathrm{~kg} / \mathrm{m}^{3}$ & $27.95 \mathrm{~kg} / \mathrm{m}^{3}(2.9 \mathrm{SDs})$ \\
\hline
\end{tabular}

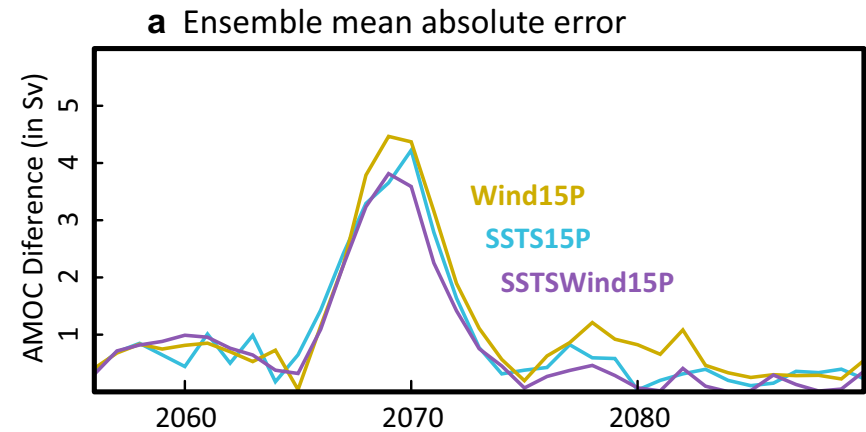

b Accumulated ensemble mean absolute error

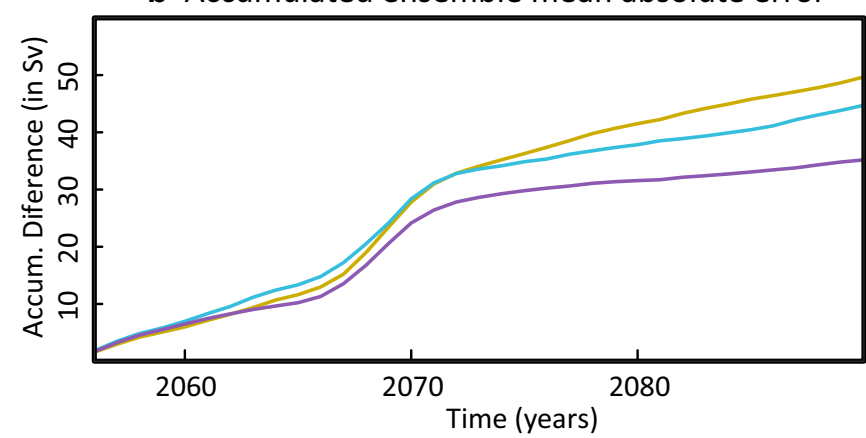

Fig. 3 a Evolution of the ensemble mean absolute error (AE) in the three nudged sets of experiments: Wind15P, SSTS15P and WindSSTS15P. Each ensemble is composed of four individual experi-

SSTSWind15P thus constitutes our baseline strategy for the new developments in Sect. 5, implemented to improve the representation of the MOI-48N extreme. But first, next section explores the causes of this extreme event and the reasons for its misrepresentation in the standard nudging experiments.

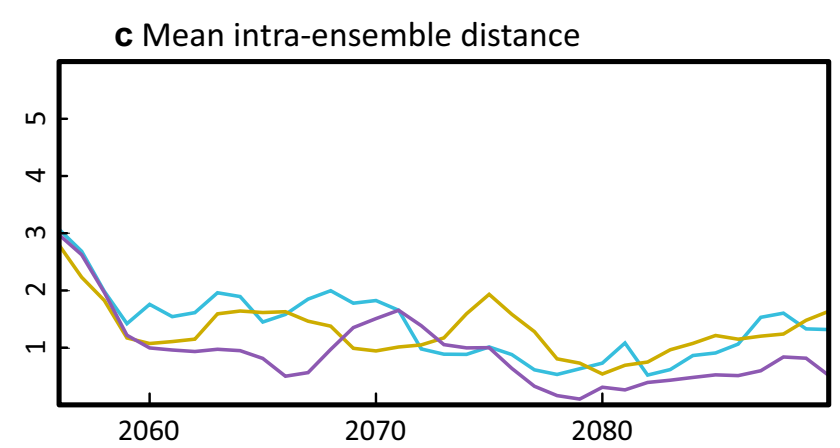

d Accumulated mean intra-ensemble distance

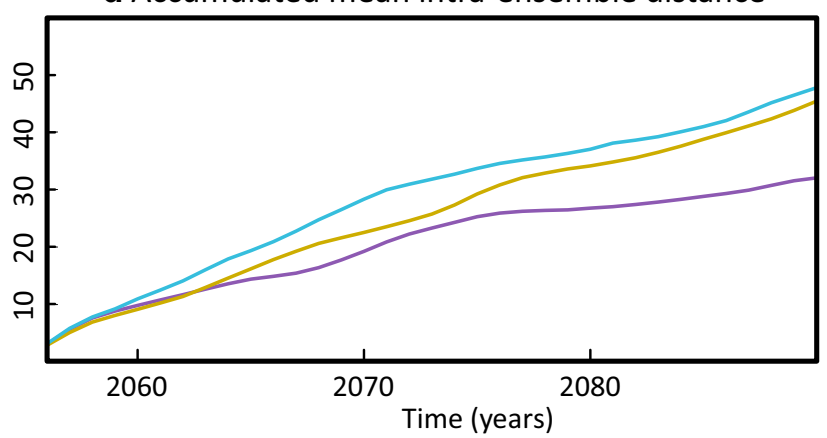

ments initialized from weak, moderate strong and exact AMOC starting conditions. b Accumulated ensemble mean absolute errors. c, d Same as in $\mathbf{a}, \mathbf{b}$ but for the mean intra-ensemble distance (IED)

\section{Causes of the AMOC peak and its misrepresentation}

To understand why the AMOC extreme occurs and why it is not well captured by the different nudging approaches, it is important to quantify the contribution of the key 
precursors of AMOC variability identified in IPSL-CM5ALR (Fig. 1) and investigate how they are initialised. We first consider the variability mechanism occurring in the upper layers of the subpolar North Atlantic described by Escudier et al. (2013). It relates to an advective mode in which Labrador salinity leads by 5 years the changes in deep convection south of Iceland (referred to as the southern convection region), and this controls the AMOC changes 6-7 years ahead. Further interactions with arctic sea-ice and sea level pressure impact the intensity of the East Greenland Current, which, 3 years later, has a negative feedback on Labrador salinity. In principle, all these surface variables can be, at least to some extent, directly reconstructed by the surface nudging, as investigated in Fig. 4. To facilitate the comparison among the different sets of nudged experiments, only the envelope and the mean of the respective ensembles are shown.

A relatively extreme minimum of the EGC strength (i.e. just below the 5th percentile from the complete 1000-year long CTRL, cf. Table 2) is detected in CTRL 15 years
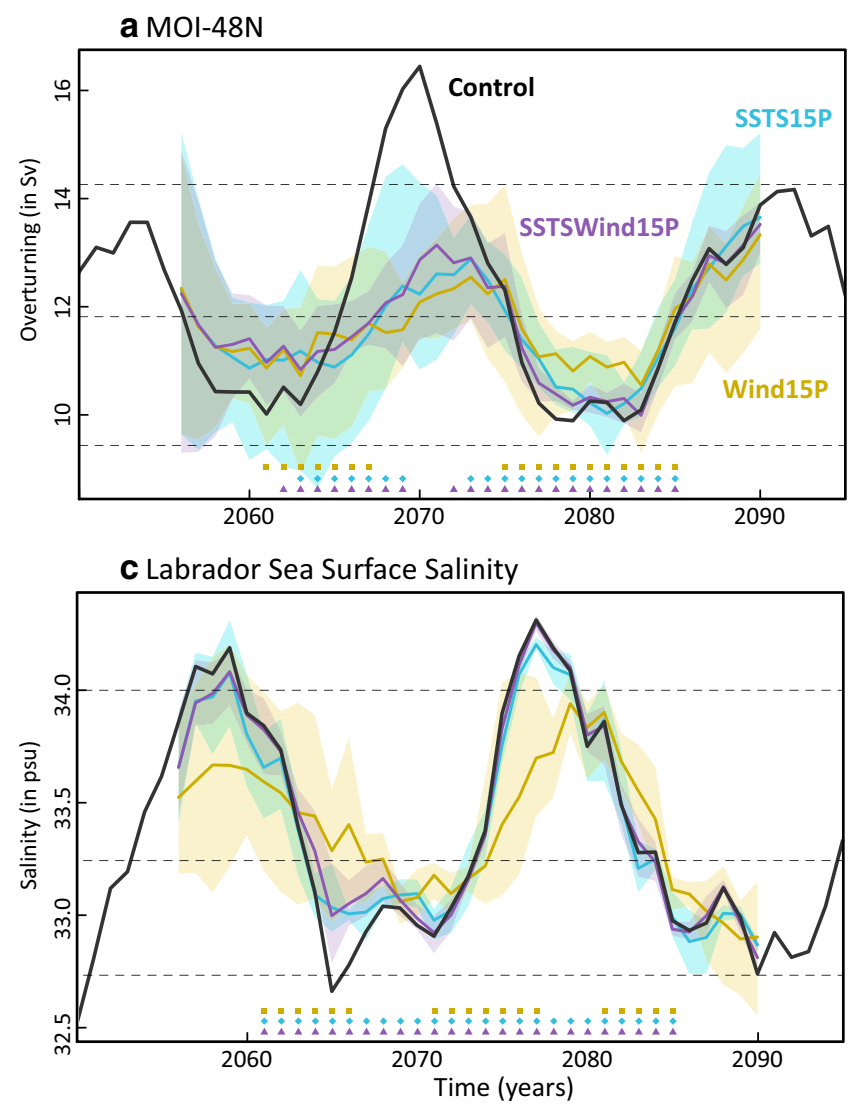

Fig. 4 a Evolution of the MOI-48N in CTRL and the nudged ensembles Wind15P, SSTS15P and SSTSWind15P. Colored envelops represent the total ensemble spread, and thick colored lines the associated ensemble mean. Horizontal dashed lines mark the climatological mean and the associated 5th and 95th percentiles of the CTRL simulation. Symbols at the bottom of each panel highlight the centre of before the peak (Fig. 4b), its magnitude being underestimated in the three nudging ensembles. The comparatively improved representation of the magnitude of other subsequent extrema (e.g. in 2066 and 2074) suggests that this issue is likely caused by the early occurrence of the EGC minimum, preventing most of the thermohaline contribution to the EGC to be properly reconstructed. Consistently with the surface mode described above, 3 years later there is a maximum in Labrador SSS in CTRL (Fig. 4c), which is well captured by the two ensembles initialising this variable (both crossing the 95th percentile), and largely underestimated in the Wind15P ensemble. This again shows that nudging wind stress alone is insufficient to represent specific thermohaline events. It is also important to note that even if both the EGC minimum (in 2056) and Labrador SSS maximum (in 2060) are respectively below/above the 5th/95th percentiles in CTRL, they are clearly not as extreme as the maximum of the MOI-48N (in 2070, see Table 2). The exceptionality of this maximum, representing 3.1 standard deviations above the MOI-48N mean,
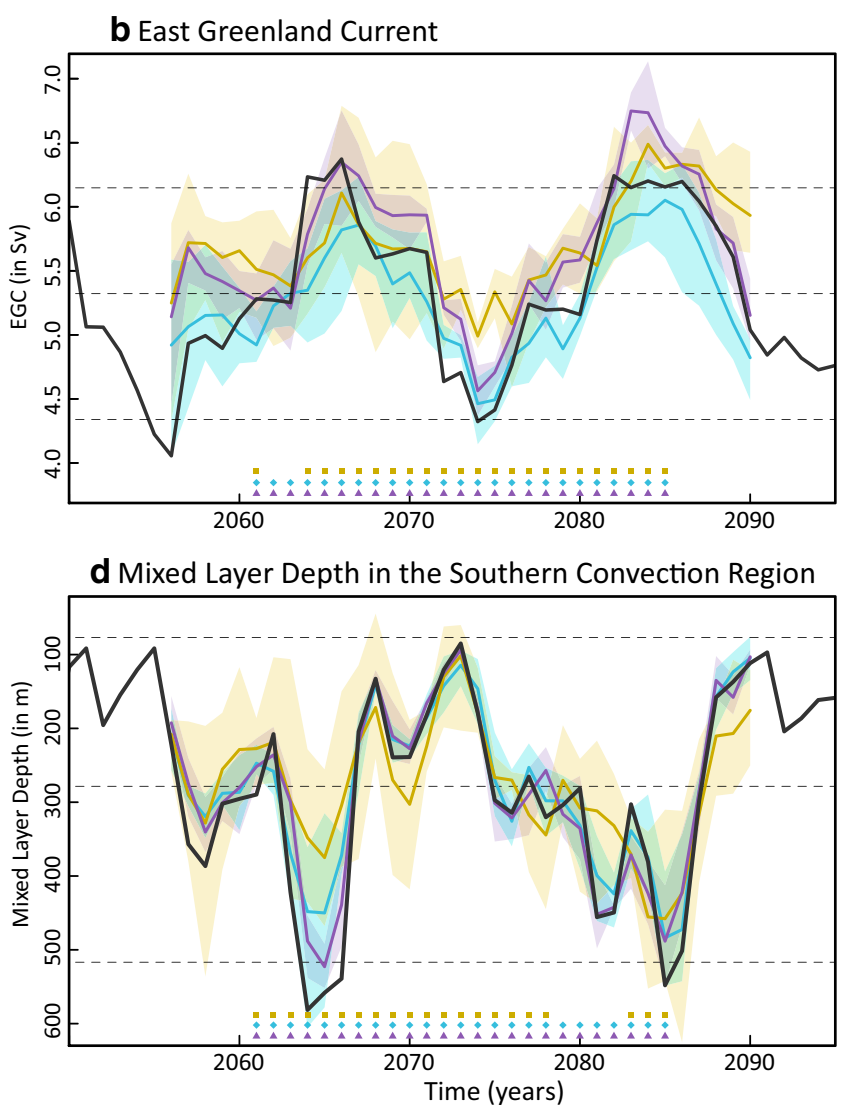

11-year long sliding windows in which the free and the nudged simulation correlate significantly at the $95 \%$ confidence level. b-d Same as in a but for the East Greenland Current, the Labrador Sea Surface Salinity and the mixed layer depth in the Southern Convection region, respectively 
can neither be explained by the previous indices, nor by the peak in the mixed layer depth (MLD) south of Iceland observed 6 years before (Fig. 4d). Note that the MLD maximum is reproduced in the three nudging ensembles, although with different degrees of accuracy (the peak is underestimated by 7,20 and 35\% in the SSTSWind15P, SSTS15P and Wind15P ensemble means, respectively).

In order to understand the extreme nature of the MOI$48 \mathrm{~N}$ peak it is essential to analyse the deeper ocean. Indeed, Ortega et al. (2015) showed that the surface mechanism in IPSL-CM5A-LR is coupled to a subsurface basin mode that helps to explain how the previous convection changes impact the AMOC. Positive density anomalies formed during convection in the top $2000 \mathrm{~m}$ induce a geostrophic response, characterized by a cyclonic flow that leads to a westward propagation of the density anomaly resulting from its interaction with the mean meridional density gradient. In this framework, the 6-7 year lead-time between convection and the AMOC corresponds to the transit time of these density anomalies to reach the western Atlantic margin, where they contribute to establish a zonal density gradient that enhances the AMOC through thermal wind balance. This zonal gradient is characterised by positive density anomalies in $300-3000 \mathrm{~m}$ at the western boundary, and concomitant negative density anomalies in 300-1000 $\mathrm{m}$ at the eastern boundary. We now analyse the reconstruction of these relevant subsurface quantities (Fig. 5).

In the southern convection region, the top $1000 \mathrm{~m}$ density anomalies in CTRL (Fig. 5a) exhibit a marked positive peak 1 year after the mixed layer is deepest, although its magnitude is less extreme than for the MOI- $48 \mathrm{~N}$ maximum (Table 2). Interestingly, a comparable extreme (i.e. 3 standard deviations above the mean) is found at deeper levels (1000-2000 m, Fig. 5b), suggesting that the characteristics of the newly formed deep waters are more important than the depth of convection itself (reasonably well represented in Fig. 4d) to explain the exceptional AMOC peak in 2070. Following the basin-mode mechanism in Ortega et al. (2015), these dense anomalies $\left(27.92 \mathrm{~kg} / \mathrm{m}^{3}\right.$ during the peak) propagate westward and penetrate slightly downwards, leading to a extreme $300-3000 \mathrm{~m}$ density peak at the western boundary in 2071 (Fig. 5c). This is preceded by the arrival of relatively light waters at the eastern side
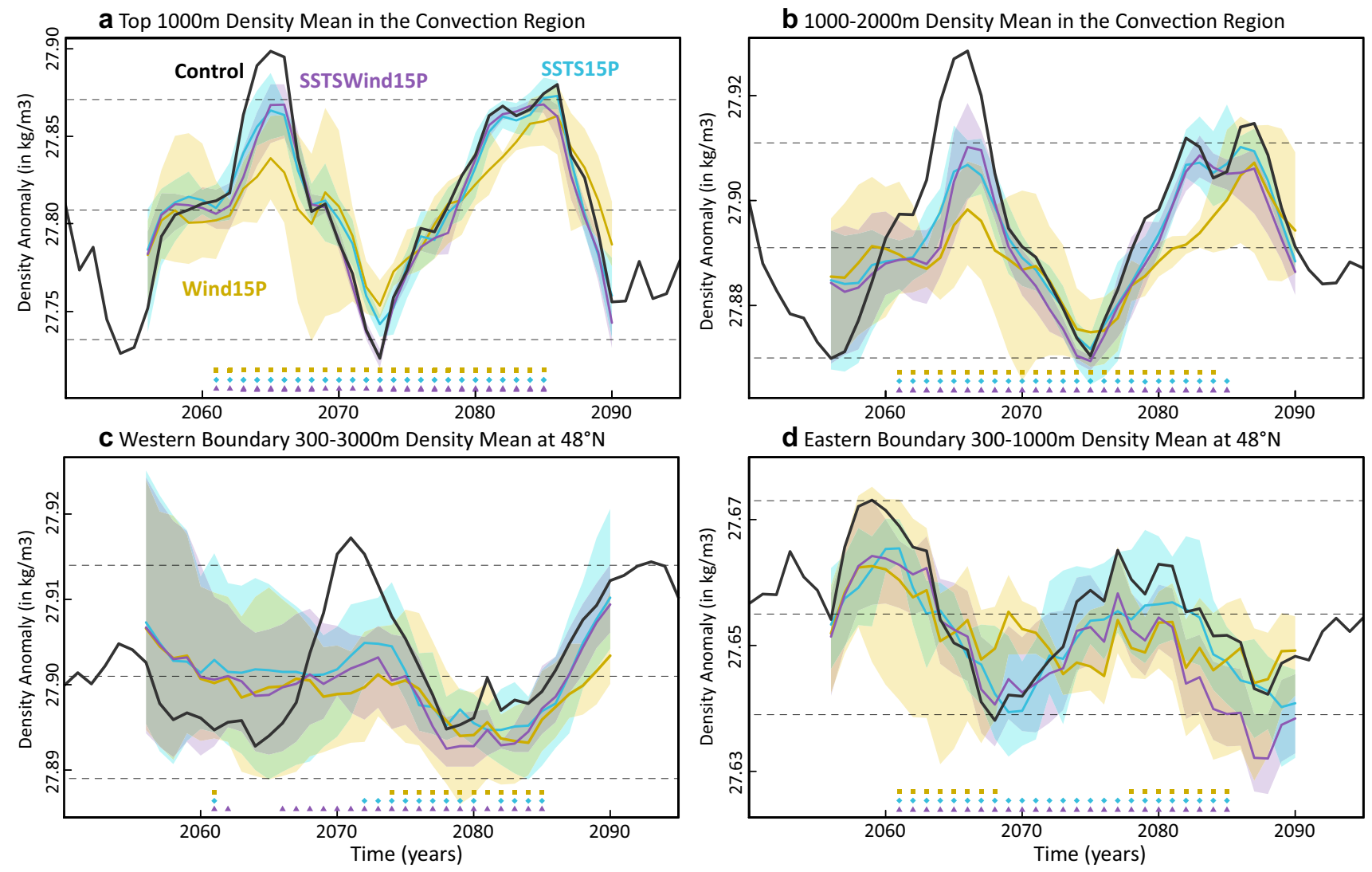

Fig. 5 Same as in Fig. 4 but for a selection of indices at depth: a, b the vertically averaged top 1000 and 1000-2000 m densities over the region of convection south of Iceland, respectively, $\mathbf{c}$ the vertically

averaged 300-3000 m densities in the western Atlantic boundary at $48^{\circ} \mathrm{N}$, and $\mathbf{d}$ the vertically averaged $300-1000 \mathrm{~m}$ densities in the eastern Atlantic boundary at $48^{\circ} \mathrm{N}$ 
in 2069 (Fig. 5d; Table 2). Thus, these concomitant signals contribute to the occurrence of the MOI- $48 \mathrm{~N}$ maximum in 2070 (Fig. 5c). In terms of exceptionality, the peak is explained by the contribution of particularly dense waters $\left(27.95 \mathrm{~kg} / \mathrm{m}^{3}, 2.9 \mathrm{SDs}\right.$ above the mean) in the deepest levels (i.e. 2000-3000 m) of the western margin (Table 2).

We now investigate the conditions leading to the exceptional dense water formation in the region of convection 5 years before the AMOC peak. Coinciding with the appearance of the dense anomalies, we identify four severe winters with remarkably negative anomalous heat fluxes (i.e. the atmosphere cooling the ocean; Fig. 6a), all below the 5th percentile from the control simulation. Their cumulative cooling effect comes on top of the arrival of positive salinity anomalies from the Labrador Sea (Fig. 4c) associated with the so-called surface mechanism. Both SST and SSS anomalies add up to produce dense anomalies and enhance convection by reducing the ocean stratification.

The reconstruction of the key anomalous heat fluxes identified above, whose origin in CTRL is presumably stochastic, is explored in a subset of the nudged experiments. Note that, in our experimental setup, heat fluxes can only be constrained indirectly through the nudging of surface temperature. For simplicity, this analysis is exclusively focused on the SSTSWind15P approach, which shows the best overall performance. Only two of the individual experiments are considered, the one started from a moderate AMOC state (as it represents starting conditions leading to a complete misrepresentation of the AMOC peak), and the experiment using exact initial conditions from CTRL (which reproduces most accurately the peak, despite the underestimation). For both cases it is evident that the evolution of heat fluxes from CTRL is not well constrained (Fig. 6b). This is particularly true for the moderate AMOC initial conditions, which show positive instead of negative heat fluxes at the time when the dense anomalies are formed. By contrast, the simulation using the exact initial state does reproduce some part of the cooling (about 25\% of the total anomaly), but not the correct intensity.

To quantify the effect of the anomalous heat fluxes on dense water formation, we perform a water mass transformation analysis for the wider North Atlantic sector $\left(20-80^{\circ} \mathrm{N}\right.$ and $\left.80^{\circ} \mathrm{W}-40^{\circ} \mathrm{E}\right)$, following Walin (1982) and Speer and Tziperman (1992). Similarly to Servonnat et al. (2015), we concentrate on the diabatic contributions of surface buoyancy forcing during the 4 years of extreme negative heat fluxes. As expected, CTRL shows a substantial increase in the formation of the densest waters $\left(28.0 \mathrm{~kg} / \mathrm{m}^{3}\right)$ during this period as compared to climatology (black line vs filled grey contour in Fig. 7a). This increase is somewhat reproduced in the SSTSWind15P simulation with exact initial conditions (blue line), but for slightly lighter waters $\left(27.95 \mathrm{~kg} / \mathrm{m}^{3}\right)$. This misrepresentation mostly comes from
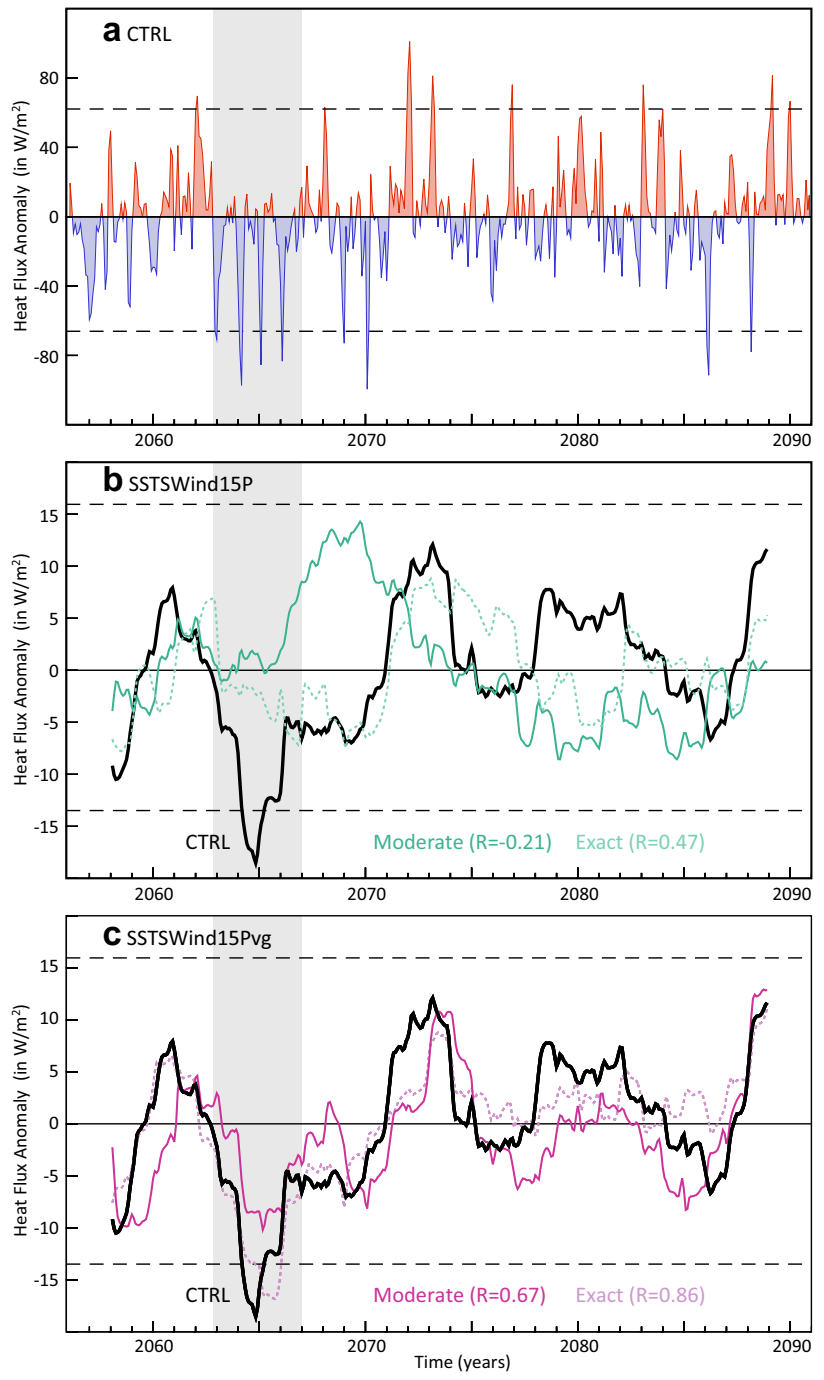

Fig. 6 a Deseasonalized monthly heat flux anomalies over the southern convection region in CTRL. Horizontal dashed lines set the 5th and 95th percentiles of the heat fluxes in the full 1000-years CTRL. The vertical grey box highlights four consecutive winters (i.e. 2063 2066) with heat fluxes below the 5th percentile. b 48-Month running means of the anomalous heat fluxes in CTRL and two SSTSWind15P and experiments starting from moderate and exact MOC ocean conditions, respectively. Anomalies are computed over the same convection region, and refer to the long-term climatological mean in the full 1000-year CTRL. Correlation coefficients between the nudged experiments and CTRL are shown in the legend. $\mathbf{c}$ Same as $\mathbf{b}$ but for the SSTSWind15Pvg experiments

differences in the contribution of heat fluxes, with freshwater fluxes playing a minor role (Fig. 7b).

Besides the important influence of surface fluxes, convection is also highly sensitive to ocean stratification. Particularly stratified conditions can prevent deep convection even if anomalous cooling occurs at the surface. Thus, the additional effect of surface salinity is essential to destabilize the water column (Swingedouw et al. 2007). One drawback of surface nudging techniques is 

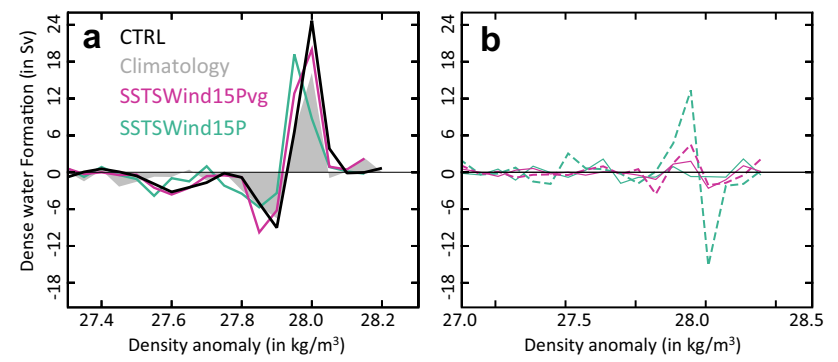

Fig. 7 a Water mass transformation (in Sv) in the North Atlantic during the period comprising the four severe winters highlighted with grey boxes in Fig. 6 . The black line represents water mass transformation in CTRL, while the dotted blue and magenta lines correspond respectively to SSTSWind15P and SSTSWind15Pvg experiments starting from the exact initial conditions in CTRL. Filled grey contours mark the 95th percentile of water mass transformation in all the possible 4-year running windows in the full 1000-years control simulation. b Difference in the mean water mass transformation with respect to CTRL due to freshwater (solid lines) and heat (dashed lines) fluxes, during the same 4 years

that they require several years to initialise the subsurface of the ocean (Servonnat et al. 2015) and thus reproduce the correct stratification. This can be a potential issue in the previous ensembles of experiments, which only begin 15 years prior to the AMOC peak (i.e. 8 before the convection peak). To investigate this, additional experiments have been performed starting the nudging procedure 25 (instead of 15) years before the peak. Figure 8a shows that, overall, the vertical density profile in these new SSTSWind25P (as compared to the previous SSTSWind15P) simulations is now better constrained (i.e. closer to the target CTRL) during the four consecutive severe winters, in particular for the experiment started from adverse initial conditions (dashed lines). Since there is more time to initialise the deeper ocean, the representation of the peak in these longer simulations is also less sensitive to the choice of initial conditions (blue lines in Fig. 9a, b). However, the extreme nature of the peak is still misrepresented due to the underestimated influence of the anomalously cold heat fluxes (not shown).

The underlying problem for this underestimation is that during deep convection events the restoring coefficient of $-40 \mathrm{~W} / \mathrm{m}^{2} / \mathrm{K}$ is too weak to correctly represent the ocean adjustment to these relatively fast atmospheric influences over the whole mixed layer depth. The next section presents a novel approach to address this issue while limiting the detrimental effects associated with the application of stronger restoring terms everywhere.
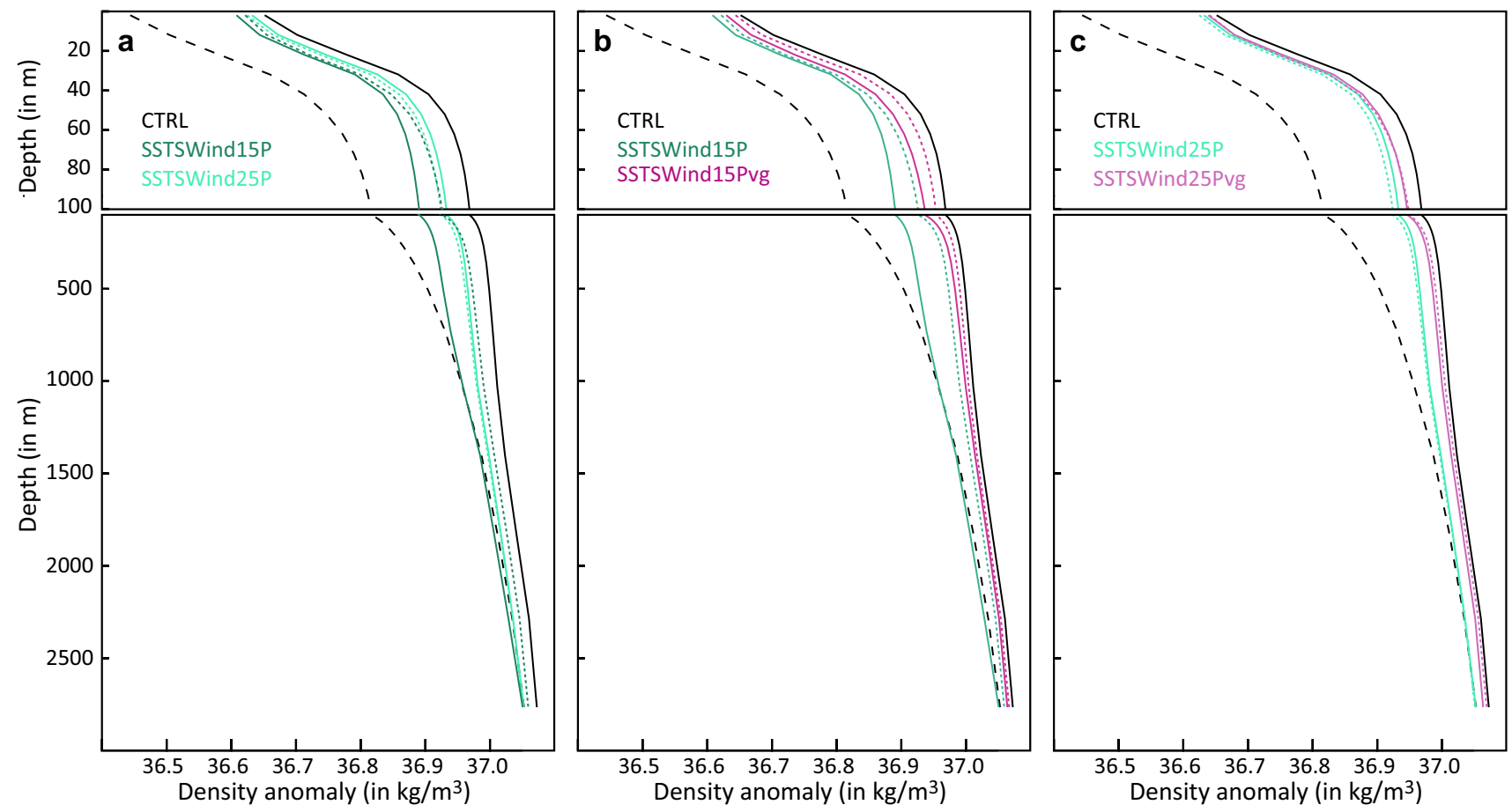

Fig. 8 Vertical profile of the mean density anomaly (sigma2) in the southern convection region during the period 2063-2066 in CTRL and different sets of nudged experiments: a SSTSWind15P and SSTSWind25P, b SSTSWind15P and SSTSWind15Pvg, and c SSTSWind25P and SSTSWind25Pvg. Full (dotted) colored lines cor- respond to experiments starting from moderate MOC (exact CTRL) ocean conditions. The black dashed lines represent to the long-term control mean and the thick black solid lines the CTRL mean during the selected period 2063-2066 

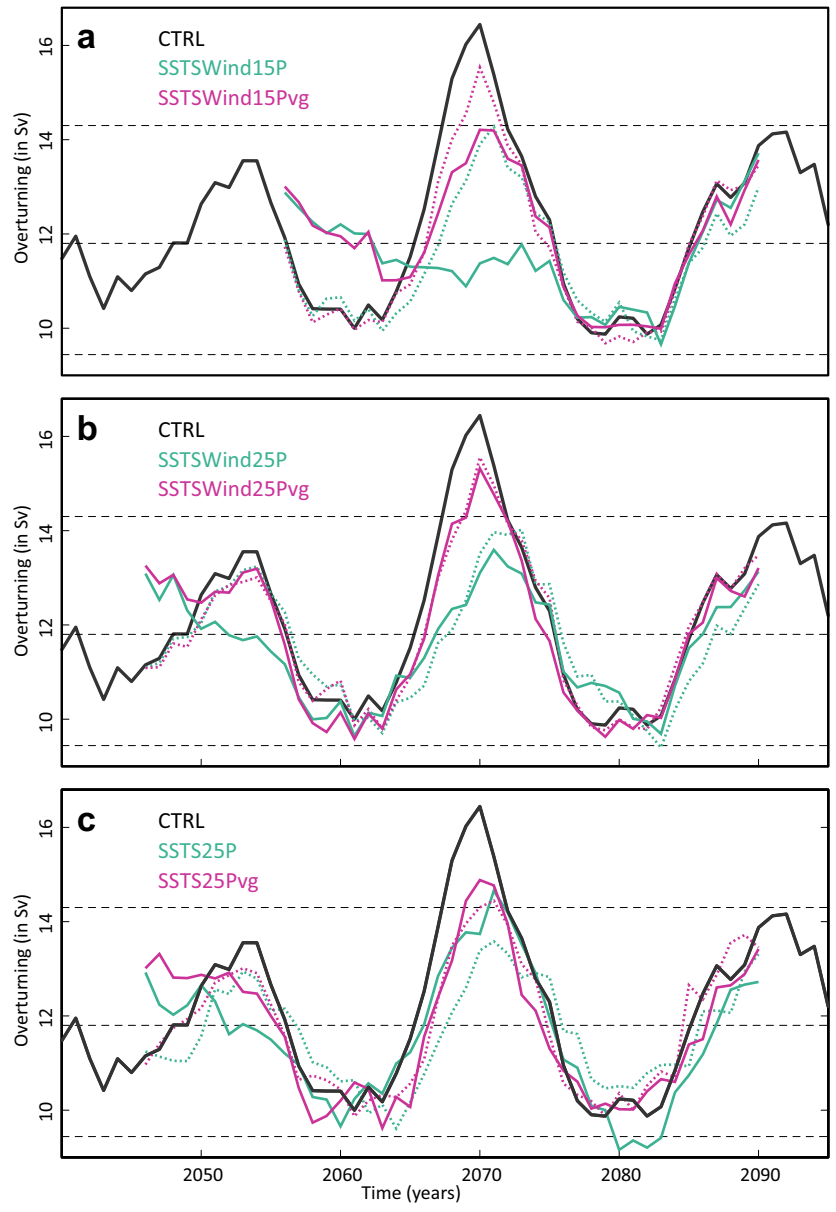

Fig. 9 Evolution of the MOI-48N-noek index in CTRL (thick black line) and both SSTSWind15P (blue lines) and SSTSWind15Pvg (magenta lines) experiments. Colored (dotted) lines account for experiments starting from moderate (exact) initial conditions. Horizontal grey dashed lines indicate the climatological mean and the 5 th and 95th percentiles of the 1000-years CTRL. b, c Same as a but for the SSTSwind25P/SSTSwind25Pvg and SSTS25P/SSTS25Pvg experiments, respectively

\section{An improved surface nudging strategy for the AMOC}

Previous sections have highlighted the importance of accurately reproducing the deep density anomalies to produce the extreme AMOC strength. As noted above, imposing the correct surface fluxes is one of the key aspects to improve. Winter deep convection typically occurs on a timescale of 2 months and involves vertical mixing at great depths $(\sim 2000 \mathrm{~m})$. However, the restoring coefficients previously applied $\left(\gamma_{\mathrm{T}}=-40 \mathrm{~W} / \mathrm{m}^{2} / \mathrm{K}, \gamma_{\mathrm{S}}=-864 \mathrm{~W} / \mathrm{m}^{2} / \mathrm{K}\right)$ are equivalent to a relaxation timescale of 60 days over a much shallower ocean layer of $50 \mathrm{~m}$. Indeed, extending the effect of restoring to the top $1000 \mathrm{~m}$ of the ocean for the same relaxation timescale would require increasing $\gamma_{\mathrm{T}}$ from -40 to $-1600 \mathrm{~W} / \mathrm{m}^{2} / \mathrm{K}$. Applying this strong $\gamma$ everywhere would most probably improve the representation of water mass transformation in some regions of the North Atlantic, but at the expense of introducing overly large correction terms in other regions where the typical mixed layer is shallower, with spurious consequences for the ocean stratification and air-sea interactions.

To overcome this limitation, we introduce a restoring term variable in time and space $\left(\gamma^{\mathrm{var}}\right)$ defined to be proportional to the mixed layer depth. In this way, the restoring strength is effectively only increased in the regions and during the months of convection, minimising the impact of unphysical correction terms elsewhere and/or in other times of the year. To make this approach directly comparable to the one based on a fixed restoring term, $\gamma^{\mathrm{var}}$ is defined so that the relaxation timescale coincides with that in the $\gamma^{\text {fix }}$ experiments. Also, $\left|\gamma^{\mathrm{fix}}\right|$ is set as the minimum absolute value that $\gamma^{\mathrm{var}}$ can achieve (i.e. $40 \mathrm{~W} / \mathrm{m}^{2} / \mathrm{K}$ for temperature and 864 for salinity) to guarantee some effective nudging in the regions where the mixed layer depth is very shallow.

By considering this latter condition, and equating the respective heat flux terms in Eq. (2) (and the freshwater terms in Eq. 3), we get:

$\frac{\gamma^{f i x}\left(S S T-S S T_{r e f}\right)}{\rho_{0} C_{p} h_{0}}=\frac{\gamma^{\mathrm{var}}\left(S S T-S S T_{r e f}\right)}{\rho_{0} C_{p} M L D}$,
$\frac{\gamma^{f i x}\left(S S S-S S S_{r e f}\right)}{h_{0}}=\frac{\gamma^{\mathrm{var}}\left(S S S-S S S_{r e f}\right)}{M L D}$,

from where it follows that,

$\gamma^{\mathrm{var}}=\max \left(\gamma^{f i x}, \gamma^{f i x} \frac{M L D}{h_{0}}\right)$,

where $h_{0}$ is $50 \mathrm{~m}$-the depth of the surface layer in the standard nudging approach-and $M L D$ the mixed layer depth in each particular grid-point. Note that this expression (Eq. 8) is valid both for the SST and SSS nudging. No changes are introduced to the wind-stress restoring approach.

This new nudging technique is applied for three different experimental setups (Table 1). First, under an equivalent configuration to SSTSWind15P, but using the variable gamma instead (SSTS15WindPvg). The second setup follows again the same procedure, but starts the simulations 25 years prior to the peak in MOI-48N (SSTSWind25Pvg), thus providing a longer time to initialise the deep ocean. Finally, in the third approach, the wind-stress restoring is removed (SSTS25Pvg), to find out if this has a detrimental effect on the final performance. The three different approaches are compared with analogous experiments using $\gamma^{\text {fix }}$. For each experiment family, two simulations are considered, corresponding to the moderate AMOC state 
and exact initial conditions from CTRL, which represent adverse and favourable conditions for the initialisation of the MOI- $48 \mathrm{~N}$ peak, respectively.

A manifest overall improvement is detected in SSTSWind15Pvg with respect to SSTSWind15P (Fig. 9a). The variable nudging allows for a better characterisation of heat flux evolution in the region of convection, evident both for the adverse and exact ocean initial conditions (Fig. 6c), the first reproducing half of the cooling anomaly in CTRL, and the second even capturing the extreme signal (i.e. below the 5 th percentile) preceding the MOI- $48 \mathrm{~N}$ peak by about 5 years. Indeed, a strong correlation $(\mathrm{R}=0.86)$ is found between the 48-month running means of the surface heat fluxes in CTRL and the SSTSWind15Pvg experiment using exact initial conditions, which is substantially higher than the correlation values for the equivalent SSTSWind15P experiment $(\mathrm{R}=0.46)$. Encouragingly, SSTSWind15Pvg also succeeds in improving the water mass transformation when compared to SSTSWind15P (Fig. 7a, b), showing, like in CTRL, a larger contribution of denser waters $\left(28.0 \mathrm{~kg} / \mathrm{m}^{3}\right.$, instead of $\left.27.9 \mathrm{~kg} / \mathrm{m}^{3}\right)$ to deep water formation. This improvement of the $\gamma^{\text {var }}$ experiments with respect to the $\gamma^{\text {fix }}$ ones is due to a better representation of both the anomalous surface heat fluxes and the target vertical stratification in the region of convection (Fig. 8b, c).

All of the above enhancements have a remarkable effect on AMOC initialisation. From Fig. 9a it is immediately clear that the simulations using $\gamma^{\text {var }}$ (magenta lines) are able to reproduce the AMOC peak, even for the adverse initial conditions. The magnitude of the peak in this latter configuration is comparable to that in the SSTSWind15P experiment using exact (and thus most favourable) initial conditions. In both cases the peak lies close to the 95th percentile in the long control simulation. Furthermore, this threshold is clearly exceeded when both exact initial conditions and $\gamma^{\mathrm{var}}$ are considered. Among all configurations investigated here, this is the first one to achieve such performance, which is the ultimate goal of the study.

Nevertheless, such an exact knowledge of the ocean initial state is not accessible in the real world. Hence, the question arises as whether it is possible to capture the

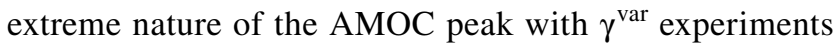
using arbitrary starting conditions. Further experiments show that it is possible, given enough time for the reconstruction. By extending the experiments to start 25 years before the AMOC peak, its extreme nature is consistently reconstructed. SSTSWind25Pvg experiments show comparable MOI-48N trajectories (magenta lines in Fig. 9b), both describing an extreme AMOC event regardless of the initial conditions considered. Additionally, we also test the contribution of the prescribed wind-stress, by reproducing the same analysis but for the SSTS25P and SSTS25Pvg experiments (Fig. 9c). The magnitude of the peak is again better represented when $\gamma^{\mathrm{var}}$ is applied, although in this case the results seem to be more sensitive to the initial state. Indeed, the AMOC extreme is better captured by the experiments initialised from a moderate AMOC ocean state (full lines in Fig. 9c) than by those using exact ocean conditions (dotted lines in Fig. 9c), like for the SSTS15P experiments in Fig. 2a.

To investigate the differences in performance for the three sets of experiments considered in this section, we first look at the generation of deep dense anomalies in the region of convection (Fig. 10a, c). In all three cases, the experiments using $\gamma^{\text {var }}$ exhibit a clear density extreme (well above the 95th percentile). It is particularly remarkable how close the SSTSWind15Pvg and SSTSWind25Pvg simulations are from the target CTRL, which confirms that a precise representation of water mass transformation is achievable when $\gamma^{\mathrm{var}}$ and wind stress restoring are combined, regardless of how close the simulation starts from the peak, or which starting conditions are considered. The remaining differences seen for the AMOC in Fig. 9 are therefore not related to the formation of deep waters by convection. Differences can emerge as these subsurface waters move westward via geostrophic self-advection (Ortega et al. 2015) and their propagation is altered by potentially unfavourable conditions in the neighbouring ocean areas-where convection is absent or shallow and the deep ocean takes longer to be reconstructed. Indeed, the dense waters reaching the western boundary and affecting the AMOC (Fig. 10d-f) are no longer as extreme as in the convection region, and show larger differences between CTRL and the nudged simulations. For example, the SSTSWind15Pvg simulations reproduce only between 60 and $70 \%$ of the western boundary positive density anomaly in CTRL. Among all the families of experiments, the largest density anomaly at this western side of the basin (87\% of CTRL) corresponds to the SSTSWind25Pvg experiment simulation using moderate AMOC initial conditions. This implies that, unlike for the MOI-45N-noek index (Fig. 9b), the representation of the western boundary densities is not improved in the simulations using exact starting conditions. This effect is counterbalanced in these specific simulations by a stronger contribution of the densities at the eastern margin (not shown), the exceptional MOI-45Nnoek peak thus resulting from the combination of both boundary signals.

To conclude, we have shown that the new proposed nudging technique based on a variable restoring coefficient $\gamma^{\text {var }}$ outperforms the previous standard procedures (using a fixed coefficient) for three different protocols (magenta vs blue lines in Figs. 9, 10). More importantly, we have identified one particular case (SSTSWind25Pvg) for which the target AMOC extreme is consistently reproduced, without 
1000-2000m Density Mean in the Convection Region
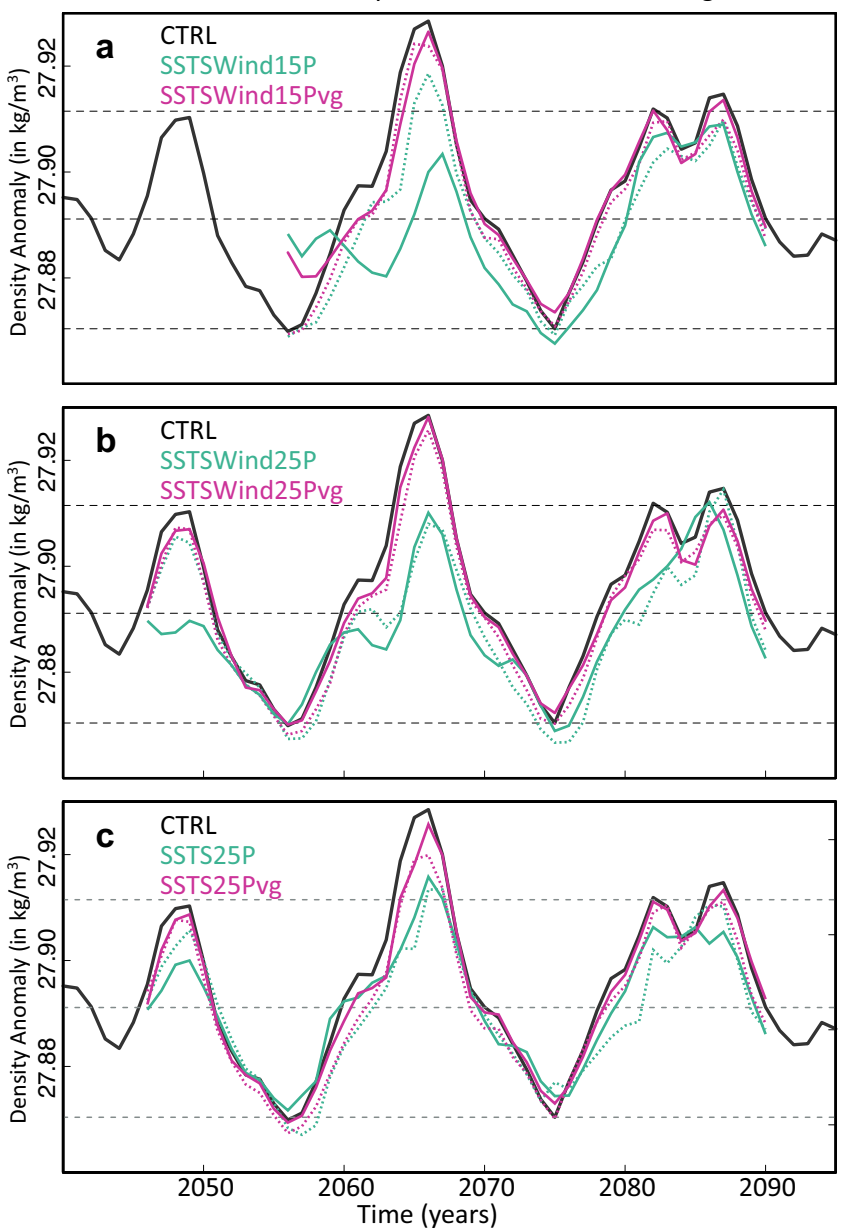

Western Boundary 300-3000m Density Mean at $48^{\circ} \mathrm{N}$
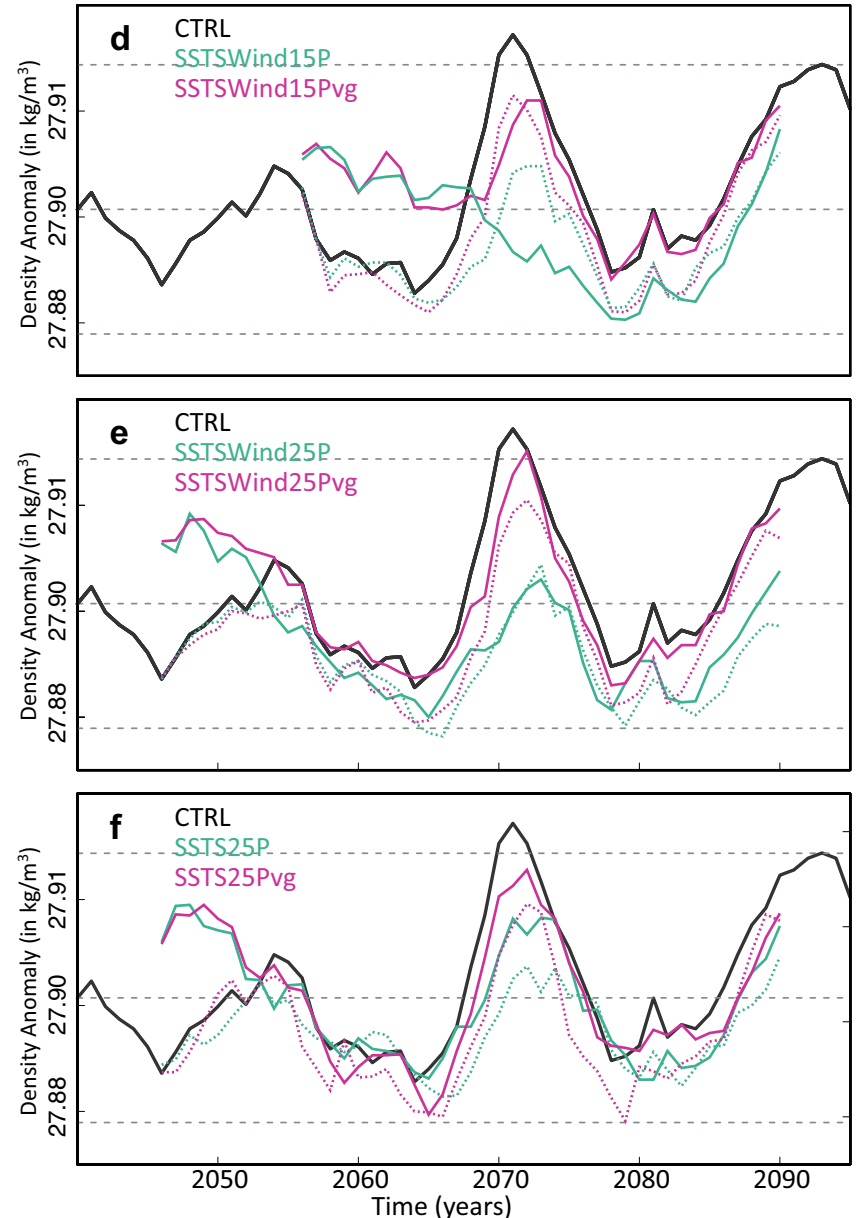

Fig. 10 Same as in Fig. 9 but for a-c the vertically averaged 1000-2000 $\mathrm{m}$ densities over the region of convection south of Iceland, and d-f the vertically averaged $300-3000 \mathrm{~m}$ densities in the western Atlantic boundary at $48^{\circ} \mathrm{N}$

requiring a perfect knowledge of the initial state of the ocean, suggesting that AMOC variability can be properly reconstructed through ocean surface nudging only.

\section{Conclusions and discussion}

Using a perfect model approach with the IPSL-CM5A-LR coupled model, we analyse how different surface nudging techniques are able to reconstruct an extreme AMOC anomaly (3 standard deviations above the long-term mean of a 1000-year long control simulation). We have shown that classical nudging protocols, using fixed physicallybased restoring terms $\left(\gamma^{\text {fix }}\right)$, can broadly reproduce the AMOC evolution from a target period in the control simulation (CTRL), but fail to capture the magnitude of the exceptional AMOC maximum. This AMOC peak is associated with the occurrence of four consecutive severe winters a few years earlier that have a cumulative cooling effect and affect water mass transformation in a major region of convection South of Greenland. The exceptionally dense anomalies formed this way are later propagated westward to the North American coast, where they enhance the AMOC via the thermal wind balance. Constraining the influence of the cold atmospheric signals on convection requires a fast relaxation timescale (2-3 months) over a deep mixed layer (1000-2000 m), which is not provided using the physically-based restoring coefficients, thus explaining why the AMOC peak is systematically underestimated in the previous nudged simulations.

One straightforward alternative is to increase the value of this $\gamma_{\mathrm{fix}}$. Yet, the benefits of applying a large $\gamma$ everywhere are likely outweighed by the associated disadvantages, which are especially important in experiments nudged with observations. For example, a strong $\gamma^{\text {fix }}$ can introduce highly unrealistic surface fluxes in regions where the model climatology deviates strongly from the observations, and jeopardise at the same time the ocean subsurface 
(by creating the wrong water mass properties) and the atmosphere (by perturbing the high-frequency air-sea interactions).

A novel approach is proposed here to overcome these limitations. By implementing a time and spatially varying restoring coefficient $\left(\gamma^{\mathrm{var}}\right)$ that is proportional to the mixed layer depth, this flexible nudging technique allows for a stronger relaxation in the areas of deep convection-which play a key role in deep water formation and thereby in the AMOC - while keeping a weak restoring term (more compatible with the observed heat-flux feedbacks) in regions and months with a shallow mixed layer. The new experiments based on this $\gamma^{\text {var }}$ show an encouraging improvement in the representation of the AMOC variability with respect to the experiments using $\gamma^{\text {fix }}$. Furthermore, for one particular setup (SSTSWind25Pvg), the timing and extreme nature of the AMOC maximum are both properly captured, irrespective of the initial conditions used. This corresponds to experiments that meet three requirements: (1) they begin 25 years before the peak, (2) nudge both sea surface temperature and salinity with $\gamma^{\mathrm{var}}$, and (3) simultaneously prescribe the wind stress field. The first requirement is important to provide sufficient time for the deep ocean (key for vertical stratification) to be correctly reconstructed. This relatively long time span associated with the surface nudging techniques is likely due to slow dynamical and thermodynamical adjustments taking place in the subsurface. Additionally, the combined restoring to the SST and SSS fields is key to successfully capture the general evolution of the AMOC. This choice was justified in a previous study (Servonnat et al. 2015) over the use of SST only nudging, which showed a poorer performance, in particular in the representation of the AMOC. Finally, the additional prescription of the wind-stress fields appears to be pivotal to both reduce the sensitivity to the initial conditions and reproduce the extreme nature of the AMOC peak. Equivalent experiments started 15 years before the AMOC maximum can still capture the general AMOC evolution, but fail to robustly reconstruct the extreme nature of the peak.

Ultimately, this study aims to identify a nudging protocol that can be applied in the historical context to faithfully constrain the past AMOC variability, and help reduce the large uncertainties among the current ocean reanalyses (Karspeck et al. 2015). The novel technique described above shows therefore a great potential, as it has been specifically conceived to improve the characterisation of the key relevant contributions to the AMOC. Furthermore, its exclusively reliance on surface data for which long observations and reanalysed atmospheric data are available (e.g. EN4 for the SST and SSS fields, and the NOAA-CIRES twentieth Century Reanalysis for the surface winds) opens the possibility of reconstructing the AMOC variability back to year 1900 AD. Note that the first 25 years in these simulations would be subject to larger uncertainties, due to the poorer quality of the observational products, and also to the long time required to initialise the deep ocean. Furthermore, the applicability of this approach to the real world is not straightforward. The first challenge relates to the combination of SST and SSS observational datasets. In perfect model studies, like the current one, SST and SSS fields are physically consistent. This means that when nudged together they both contribute to constrain the correct density fields. In the real world, by contrast, beyond the fact that salinity observations are far less numerous and reliable, temperature and salinity observations can present large uncertainties introducing inconsistencies between the two fields. Some preliminary tests show that these potentially conflicting signals can substantially damp AMOC variability. A second important issue relates to the fact that the AMOC is highly model-dependent, and sensitive to model biases. As a result, the drivers of the AMOC can largely vary from one model to another. Indeed, the IPSLCM5A-LR model analysed here presents a strong negative salinity bias that prevents the simulation of deep convection in the Labrador Sea (Escudier et al. 2013), which is a major region of deep water formation in other models and also in the real world. The performance of the $\gamma^{\mathrm{var}}$ nudging to reconstruct the AMOC therefore has to be evaluated in other models with different AMOC variability. And another key question is what will be the effect of the model biases when nudging to the observed variability. These issues are currently addressed and will be reported in a follow-up study. Further modifications to the current protocol, such as prescribing the full $3 \mathrm{D}$ wind structure, and the nudging towards surface temperature over the continents are also envisaged for future studies.

Acknowledgements This study was partly funded by the EU project SPECS funded by the European Commission's Seventh Framework Research Programme under the Grant agreement 308378, and by the MORDICUS Project funded by the Agence Nationale de la Recherche under Grant agreement ANR-13-SENV-0002-02. This work was granted access to the HPC resources of TGCC under the allocation gen7403 made by GENCI. The authors thank two anonymous reviewers for their helpful comments and suggestions.

Open Access This article is distributed under the terms of the Creative Commons Attribution 4.0 International License (http:// creativecommons.org/licenses/by/4.0/), which permits unrestricted use, distribution, and reproduction in any medium, provided you give appropriate credit to the original author(s) and the source, provide a link to the Creative Commons license, and indicate if changes were made.

\section{References}

Allison L, Hawkins E, Woollings T (2014) An event-based approach to understanding decadal fluctuations in the Atlantic meridional 
overturning circulation. Clim Dyn 44:163-190. doi:10.1007/ s00382-014-2271-9

Aumont O, Bopp L (2006) Globalizing results from ocean in situ iron fertilization studies. Glob Biogeochem Cycles. doi:10.1029/200 5GB002591

Balmaseda MA, Hernandez F, Storto A et al (2015) The ocean reanalyses intercomparison project (ORA-IP). J Oper Oceanogr 8:s80 s97. doi:10.1080/1755876X.2015.1022329

Barnier B, Siefridt L, Marchesiello P (1995) Thermal forcing for a global ocean circulation model using a 3-year climatology of ECMWF analyses. J Mar Syst 6:363-380. doi:10.1016/0924-7963(94)00034-9

Boer GJ (2004) Long time-scale potential predictability in an ensemble of coupled climate models. Clim Dyn 23:29-44. doi:10.1007/ s00382-004-0419-8

Born A, Mignot J, Stocker TF (2015) Multiple equilibria as a possible mechanism for decadal variability in the North Atlantic Ocean. J Clim 28:8907-8922. doi:10.1175/JCLI-D-14-00813.1

Caya A, Buehner M, Carrieres T (2010) Analysis and forecasting of sea ice conditions with three-dimensional variational data assimilation and a coupled ice-ocean model. J Atmos Ocean Technol 27:353-369. doi:10.1175/2009JTECHO701.1

Collins M, Botzet M, Carril AF et al (2006) Interannual to decadal climate predictability in the North Atlantic: a multimodelensemble study. J Clim 19:1195-1203. doi:10.1175/JCLI3654.1

Counillon F, Bethke I, Keenlyside N et al (2014) Seasonal-to-decadal predictions with the ensemble kalman filter and the Norwegian earth System Model: a twin experiment. Tellus Ser A Dyn Meteorol Oceanogr 66:1-21. doi:10.3402/tellusa.v66.21074

Cunningham S, Kanzow T, Rayner D, et al (2007) Temporal variability of the Atlantic meridional overturning circulation at $26.5 \mathrm{~N}$. Science 317(80):935-938. doi:10.1126/science.1141304

Delworth T, Manabe S, Stouffer RJ (1993) Interdecadal variations of the thermohaline circulation in a coupled ocean-atmosphere model. J Clim 6:1993-2011. doi:10.1175/1520-0442(1993)006<1993:IVOTTC > 2.0.CO;2

Ding H, Greatbatch RJ, Latif M et al (2013) Hindcast of the 1976/77 and 1998/99 climate shifts in the Pacific. J Clim 26:7650-7661. doi:10.1175/JCLI-D-12-00626.1

Duchez A, Hirschi JJ-M, Blaker AT et al (2014) A new index for the atlantic meridional overturning circulation at $26^{\circ} \mathrm{N}$. J Clim 27:140519141100008. doi:10.1175/JCLI-D-13-00052.1

Duchez A, Courtois P, Harris E et al (2016) Potential for seasonal prediction of Atlantic sea surface temperatures using the RAPID array at $26^{\circ} \mathrm{N}$. Clim Dyn 46:3351-3370. doi:10.1007/ s00382-015-2918-1

Dufresne JL, Foujols MA, Denvil S et al (2013) Climate change projections using the IPSL-CM5 earth system model: from CMIP3 to CMIP5. Clim Dyn 40:2123-2165. doi:10.1007/ s00382-012-1636-1

Dunstone NJ, Smith DM (2010) Impact of atmosphere and sub-Surface ocean data on decadal climate prediction. Geophys Res Lett 37:1-5. doi:10.1029/2009GL041609

Escudier R, Mignot J, Swingedouw D (2013) A 20-year coupled ocean-sea ice-atmosphere variability mode in the North Atlantic in an AOGCM. Clim Dyn 40:619-636. doi:10.1007/ s00382-012-1402-4

Fichefet T, Maqueda MAM (1997) Sensitivity of a global sea ice model to the treatment of ice thermodynamics and dynamics. J Geophys Res Ocean 102:12609-12646. doi:10.1029/97JC00480

Frajka-Williams E (2015) Estimating the Atlantic overturning at 26??N using satellite altimetry and cable measurements. Geophys Res Lett 42:3458-3464. doi:10.1002/2015GL063220

Frankignoul C, Kestenare E (2002) The surface heat flux feedback. Part I: estimates from observations in the Atlantic and the North Pacific. Clim Dyn 19:633-647. doi:10.1007/s00382-002-0252-x
Han E, Merwade V, Heathman GC (2012) Implementation of surface soil moisture data assimilation with watershed scale distributed hydrological model. J Hydrol 416-417:98-117. doi:10.1016/j.jhydrol.2011.11.039

Hourdin F, Foujols MA, Codron F et al (2013) Impact of the LMDZ atmospheric grid configuration on the climate and sensitivity of the IPSL-CM5A coupled model. Clim Dyn 40:2167-2192. doi:10.1007/s00382-012-1411-3

Huck T, de Verdière A, Estrade P, Schopp R (2008) Low-frequency variations of the large-scale ocean circulation and heat transport in the North Atlantic from 1955 to 1998 in situ temperature and salinity data. Geophys Res Lett 35:L23613. doi:10.10 29/2008GL035635

Karspeck AR, Stammer D, Köhl A, et al (2015) Comparison of the Atlantic meridional overturning circulation between 1960 and 2007 in six ocean reanalysis products. Clim Dyn. doi:10.1007/ s00382-015-2787-7

Kissel C, Van Toer A, Laj C et al (2013) Variations in the strength of the North Atlantic bottom water during Holocene. Earth Planet Sci Lett 369-370:248-259. doi:10.1016/j. eps1.2013.03.042

Krinner G, Viovy N, de Noblet-Ducoudré N et al (2005) A dynamic global vegetation model for studies of the coupled atmospherebiosphere system. Glob Biogeochem Cycles 19:GB1015 doi:10 $.1029 / 2003 \mathrm{gb} 002199$

Large WG, Yeager SG (2009) The global climatology of an interannually varying air-sea flux data set. Clim Dyn 33:341-364. doi:10.1007/s00382-008-0441-3

Latif M, Roeckner E, Botzet $M$ et al (2004) Reconstructing, monitoring, and predicting multidecadal-scale changes in the North Atlantic thermohaline circulation with sea surface temperature. J Clim 17:1605-1614. doi:10.1175/1520-0442(2004)017<1605:RMAPMC > 2.0.CO;2

Luo JJ, Masson S, Behera S et al (2005) Seasonal climate predictability in a coupled OAGCM using a different approach for ensemble forecasts. J Clim 18:4474-4497. doi:10.1175/JCLI3526.1

Madec G (2008) NEMO ocean engine, Technical Note, IPSL

Marotzke J, Giering R, Zhang KQ et al (1999) Construction of the adjoint MIT ocean general circulation model and application to Atlantic heat transport sensitivity. J Geophys Res 104547:529529. doi:10.1029/1999JC900236

Matei D, Baehr J, Jungclaus JH, et al (2012) Multiyear prediction of monthly mean Atlantic meridional overturning circulation at $26.5^{\circ} \mathrm{N}$. Science 335 (80):76-79. doi:10.1126/science. 1210299

McCarthy GD, Haigh ID, Hirschi JJ-M et al (2015) Ocean impact on decadal Atlantic climate variability revealed by sea-level observations. Nature 521:508-510. doi:10.1038/nature14491

McManus JF, Francois R, Gherardi J-M et al (2004) Collapse and rapid resumption of Atlantic meridional circulation linked to deglacial climate changes. Nature 428:834-837. doi:10.1038/ nature 02494

Meehl GA, Goddard L, Boer G et al (2014) Decadal climate prediction an update from the trenches. Bull Am Meteorol Soc 95:243267. doi:10.1175/BAMS-D-12-00241.1

Mercier H, Lherminier P, Sarafanov A et al (2015) Variability of the meridional overturning circulation at the Greenland-Portugal OVIDE section from 1993 to 2010. Prog Oceanogr 132:250 261. doi:10.1016/j.pocean.2013.11.001

Mignot J, Swingedouw D, Deshayes J et al (2013) On the evolution of the oceanic component of the IPSL climate models from CMIP3 to CMIP5: a mean state comparison. Ocean Model 72:167-184. doi:10.1016/j.ocemod.2013.09.001

Mignot J, García-Serrano J, Swingedouw D et al (2015) Decadal prediction skill in the ocean with surface nudging in the IPSLCM5A-LR climate model. Clim Dyn 1-22. doi:10.1007/ s00382-015-2898-1 
Msadek R, Dixon KW, Delworth TL, Hurlin W (2010) Assessing the predictability of the Atlantic meridional overturning circulation and associated fingerprints. Geophys Res Lett 37:L19608. doi:10 .1029/2010GL044517

Ortega P, Hawkins E, Sutton R (2011) Processes governing the predictability of the Atlantic meridional overturning circulation in a coupled GCM. Clim Dyn 37:1771-1782. doi:10.1007/ s00382-011-1025-1

Ortega P, Mignot J, Swingedouw D et al (2015) Reconciling two alternative mechanisms behind bi-decadal variability in the North Atlantic. Prog Oceanogr 137:237-249. doi:10.1016/j. pocean.2015.06.009

Persechino A, Mignot J, Swingedouw D et al (2013) Decadal predictability of the Atlantic meridional overturning circulation and climate in the IPSL-CM5A-LR model. Clim Dyn 40:2359-2380. doi:10.1007/s00382-012-1466-1

Pohlmann H, Jungclaus JH, Köhl A et al (2009) Initializing decadal climate predictions with the GECCO oceanic synthesis: effects on the North Atlantic. J Clim 22:3926-3938. doi:10.1175/2009 JCLI2535.1

Ray S, Swingedouw D, Mignot J, Guilyardi E (2015) Effect of surface restoring on subsurface variability in a climate model during 1949-2005. Clim Dyn 44:2333-2349. doi:10.1007/ s00382-014-2358-3

Reichler T, Kim J, Manzini E, Kroger J (2012) A stratospheric connection to Atlantic climate variability. Nat Geosci 5:783-787

Robson J, Hodson D, Hawkins E, Sutton R (2014) Atlantic overturning in decline? Nat Geosci 7:2-3. doi:10.1038/ngeo2050

Servonnat J, Mignot J, Guilyardi E et al (2015) Reconstructing the subsurface ocean decadal variability using surface nudging in a perfect model framework. Clim Dyn 44:315-338. doi:10.1007/ s00382-014-2184-7

Sévellec F, Fedorov AV (2013) The leading, interdecadal eigenmode of the Atlantic Meridional overturning circulation in a realistic ocean model. J Clim 26:2160-2183. doi:10.1175/ JCLI-D-11-00023.1

Shaffrey LC, Hodson D, Robson J et al (2016) Decadal predictions with the HiGEM high resolution global coupled climate model: description and basic evaluation. Clim Dyn. doi:10.1007/ s00382-016-3075-x

Smeed DA, McCarthy GD, Cunningham SA et al (2014) Observed decline of the Atlantic meridional overturning circulation 20042012. Ocean Sci 10:29-38. doi:10.5194/os-10-29-2014

Speer K, Tziperman E (1992) Rates of water mass formation in the North Atlantic Ocean. J Phys Oceanogr 22:93-104. doi:10.1175/1520-0485(1992)022<0093:ROWMFI > 2.0.CO;2

Stewart KD, Haine TWN (2013) Wind-driven Arctic freshwater anomalies. Geophys Res Lett 40:6196-6201. doi:10.1002/201 3GL058247
Stommel HM (1961) Thermohaline convection with two stable regimes of flow. Tellus 13:224-230. doi:10.1111/j.2153-3490.1961.tb00079.x

Swingedouw D, Braconnot P, Delecluse P et al (2007) The impact of global freshwater forcing on the thermohaline circulation: adjustment of North Atlantic convection sites in a CGCM. Clim Dyn 28:291-305. doi:10.1007/s00382-006-0171-3

Swingedouw D, Mignot J, Labetoulle S et al (2013) Initialisation and predictability of the AMOC over the last 50 years in a climate model. Clim Dyn 40:2381-2399. doi:10.1007/ s00382-012-1516-8

Tatebe H, Ishii M, Mochizuki T et al (2012) The initialization of the MIROC climate models with hydrographic data assimilation for decadal prediction. J Meteorol Soc Jpn 90A:275-294. doi:10.2151/jmsj.2012-A14

Thoma M, Gerdes R, Greatbatch RJ, Ding H (2015) Partially coupled spin-up of the MPI-ESM: implementation and first results. Geosci Model Dev 8:51-68. doi:10.5194/gmd-8-51-2015

Thornalley DJR, Blaschek M, Davies FJ, et al (2013) Long-term variations in Iceland-Scotland overflow strength during the Holocene. Clim Past 9:2073-2084. doi:10.5194/cp-9-2073-2013

Turpin V, Remy E, Le Traon PY (2016) How essential are Argo observations to constrain a global ocean data assimilation system? Ocean Sci 12:257-274. doi:10.5194/osd-12-1145-2015

Vecchi G a, Msadek R, Delworth TL et al (2012) Comment on multiyear prediction of monthly mean atlantic meridional overturning circulation at $26.5 \mathrm{~N}^{\prime \prime}$. Science $338: 604 \mathrm{c}$. doi:10.1126/ science. 1222566

Walin G (1982) On the relation between sea-surface heat flow and thermal circulation in the ocean. Tellus A. doi:10.3402/tellusa. v34i2.10801

Willis JK (2010) Can in situ floats and satellite altimeters detect longterm changes in Atlantic Ocean overturning? Geophys Res Lett 37:1-5. doi:10.1029/2010GL042372

Yang X, Rosati A, Zhang S et al (2013) A predictable AMO-like pattern in the GFDL fully coupled ensemble initialization and decadal forecasting system. J Clim 26:650-661. doi:10.1175/ JCLI-D-12-00231.1

Yeager S, Karspeck A, Danabasoglu G et al (2012) A decadal prediction case study: late twentieth-century north Atlantic Ocean heat content. J Clim 25:5173-5189. doi:10.1175/JCLI-D-11-00595.1

Zhang R (2008) Coherent surface-subsurface fingerprint of the Atlantic meridional overturning circulation. Geophys Res Lett 35:L20705. doi:10.1029/2008GL035463

Zhang S, Rosati A, Delworth T (2010) The adequacy of observing systems in monitoring the Atlantic meridional overturning circulation and North Atlantic climate. J Clim 23:5311-5324. doi:10. 1175/2010JCLI3677.1 